\title{
CLOSED IDEALS OF OPERATORS ON THE TSIRELSON AND SCHREIER SPACES
}

\author{
KEVIN BEANLAND, TOMASZ KANIA, AND NIELS JAKOB LAUSTSEN
}

\begin{abstract}
Let $\mathscr{B}(X)$ denote the Banach algebra of bounded operators on $X$, where $X$ is either Tsirelson's Banach space or the Schreier space of order $n$ for some $n \in \mathbb{N}$. We show that the lattice of closed ideals of $\mathscr{B}(X)$ has a very rich structure; in particular $\mathscr{B}(X)$ contains at least continuum many maximal ideals.

Our approach is to study the closed ideals generated by the basis projections. Indeed, the unit vector basis is an unconditional basis for each of the above spaces, so there is a basis projection $P_{N} \in \mathscr{B}(X)$ corresponding to each non-empty subset $N$ of $\mathbb{N}$. A closed ideal of $\mathscr{B}(X)$ is spatial if it is generated by $P_{N}$ for some $N$. We can now state our main conclusions as follows:

- the family of spatial ideals lying strictly between the ideal of compact operators and $\mathscr{B}(X)$ is non-empty and has no minimal or maximal elements;

- for each pair $\mathscr{I} \varsubsetneqq \mathscr{J}$ of spatial ideals, there is a family $\left\{\Gamma_{L}: L \in \Delta\right\}$, where the index set $\Delta$ has the cardinality of the continuum, such that $\Gamma_{L}$ is an uncountable chain of spatial ideals, $\bigcup \Gamma_{L}$ is a closed ideal that is not spatial, and

$$
\mathscr{I} \varsubsetneqq \mathscr{L} \varsubsetneqq \mathscr{J} \quad \text { and } \quad \overline{\mathscr{L}+\mathscr{M}}=\mathscr{J}
$$

whenever $L, M \in \Delta$ are distinct and $\mathscr{L} \in \Gamma_{L}, \mathscr{M} \in \Gamma_{M}$.
\end{abstract}

\section{InTRODUCTION AND STATEMENT OF MAIN RESUltS}

Let $X$ be a Banach space with an unconditional basis $\left(b_{j}\right)_{j \in \mathbb{N}}$. For a subset $N$ of $\mathbb{N}$, we write $P_{N}$ for the basis projection corresponding to $N$; that is, $P_{N} x=\sum_{j \in N}\left\langle x, b_{j}^{*}\right\rangle b_{j}$ for each $x \in X$, where $b_{j}^{*} \in X^{*}$ denotes the $j^{\text {th }}$ coordinate functional. By a spatial ideal of the Banach algebra $\mathscr{B}(X)$ of bounded operators on $X$, we understand the closed, two-sided ideal generated by the basis projection $P_{N}$ for some non-empty subset $N$ of $\mathbb{N}$. A spatial ideal $\mathscr{I}$ is non-trivial if

$$
\mathscr{K}(X) \varsubsetneqq \mathscr{I} \varsubsetneqq \mathscr{B}(X)
$$

where $\mathscr{K}(X)$ denotes the ideal of compact operators. A chain of spatial ideals is a nonempty set $\Gamma$ of spatial ideals of $\mathscr{B}(X)$ such that $\Gamma$ is totally ordered by inclusion.

We shall study two (classes of) Banach spaces, namely Tsirelson's space on the one hand and the Schreier spaces of finite order on the other. We refer to Section 4 for details of the definition of the latter spaces, originally due to Alspach and Argyros [1]. These Banach spaces have unconditional bases. Using spatial ideals, we shall show that their lattices of

2010 Mathematics Subject Classification. 46H10, 47L10, (primary); 46B03, 46B45, 47L20.

Key words and phrases. Banach space, Tsirelson space, Schreier space, bounded operator, closed operator ideal, ideal lattice. 
closed operator ideals have a very rich structure. The following theorem summarizes our main findings.

Theorem 1.1. Let $X$ denote either Tsirelson's space $T$ or the Schreier space $X\left[\mathcal{S}_{n}\right]$ of order $n$ for some $n \in \mathbb{N}$.

(i) The family of non-trivial spatial ideals of $\mathscr{B}(X)$ is non-empty and has no minimal or maximal elements.

(ii) Let $\mathscr{I} \varsubsetneqq \mathscr{J}$ be spatial ideals of $\mathscr{B}(X)$. Then there is a family $\left\{\Gamma_{L}: L \in \Delta\right\}$ such that:

- the index set $\Delta$ has the cardinality of the continuum;

- for each $L \in \Delta, \Gamma_{L}$ is an uncountable chain of spatial ideals of $\mathscr{B}(X)$ such that

$$
\mathscr{I} \varsubsetneqq \mathscr{L} \varsubsetneqq \mathscr{J} \quad\left(\mathscr{L} \in \Gamma_{L}\right),
$$

and $\bigcup \Gamma_{L}$ is a closed ideal that is not spatial;

- $\overline{\mathscr{L}+\mathscr{M}}=\mathscr{J}$ whenever $\mathscr{L} \in \Gamma_{L}$ and $\mathscr{M} \in \Gamma_{M}$ for distinct $L, M \in \Delta$.

(iii) The Banach algebra $\mathscr{B}(X)$ contains at least continuum many maximal ideals.

We shall also consider the "small" ideals of operators on the above spaces, where we call an ideal "small" if it contains no projections with infinite-dimensional image. The particular ideals that we are interested in are the compact, strictly singular and inessential operators; we refer to Definition 2.2 for the precise definitions of the latter two operator ideals, which we denote by $\mathscr{S}$ and $\mathscr{E}$, respectively.

Theorem 1.2. $\quad$ (i) The ideals of compact, strictly singular and inessential operators on Tsirelson's space coincide, and they are equal to the intersection of the non-trivial spatial ideals of $\mathscr{B}(T)$ :

$$
\mathscr{K}(T)=\mathscr{S}(T)=\mathscr{E}(T)=\bigcap\{\mathscr{I}: \mathscr{I} \text { is a non-trivial spatial ideal of } \mathscr{B}(T)\} .
$$

(ii) Let $X=X\left[\mathcal{S}_{n}\right]$ be the Schreier space of order $n$ for some $n \in \mathbb{N}$. Then

$$
\mathscr{K}(X) \varsubsetneqq \mathscr{S}(X)=\mathscr{E}(X)
$$

and

$$
\bigcap\{\mathscr{I}: \mathscr{I} \text { is a non-trivial spatial ideal of } \mathscr{B}(X)\} \nsubseteq \mathscr{S}(X) \text {. }
$$

The paper is organized as follows: we conclude this introduction with a brief survey of related results to provide some background for our work and put it in context. In Section 2, we set up notation and establish some basic general results, as well as a common framework for the proof of Theorem 1.1, before we complete the proofs for Tsirelson's space in Section 3 and for the Schreier spaces in Section 4. Finally, Section 5 contains some open questions related to this work.

The seminal study of operator ideals is due to Calkin [5], who considered the situation when the underlying Banach space is a separable Hilbert space. His most important conclusion (at least from our point of view) is that the ideal of compact operators is the only 
proper, non-zero closed ideal in this case. Gohberg, Markus and Feldman [15] generalized Calkin's result to the other classical sequence spaces $c_{0}$ and $\ell_{p}$ for $p \in[1, \infty)$, while Gramsch [17] and Luft [28] independently classified all the closed ideals of $\mathscr{B}(H)$ when $H$ is a non-separable Hilbert space. Their result implies that these ideals form a well-ordered chain whose length is determined by the dimension of $H$.

Berkson and Porta [4, Section 5] initiated the study of the closed ideals of $\mathscr{B}\left(L_{p}[0,1]\right)$ for $p \in(1,2) \cup(2, \infty)$, proving in particular that they are not totally ordered. Porta [35] then went on to construct a Banach space $X$ such that there is an injective map from the set $[\mathbb{N}]^{<\infty}$ of all finite subsets of the natural numbers into the lattice of closed ideals of $\mathscr{B}(X)$, and this map preserves inclusions in both directions. Porta's Banach space $X$ is the $\ell_{2}$-sum of a family of the form $\left\{\ell_{p}: p \in \mathrm{P}\right\}$ for a countably infinite subset $\mathrm{P}$ of $[1, \infty)$. By ensuring that $1 \notin \mathrm{P}$ and that $\mathrm{P}$ contains the conjugate index of each of its elements, Porta arranged that $X$ is reflexive and isometric to its dual space $X^{*}$. As far as we know, this was the first example of a separable Banach space which has infinitely many closed operator ideals.

Porta [36] also initiated the study of the closed operator ideals on $\ell_{p} \oplus \ell_{q}$ for distinct $p, q \in(1, \infty)$, notably showing that for $p=2$, there are exactly two maximal ideals, which correspond to the operators that factor through $\ell_{2}$ and $\ell_{q}$, respectively. Subsequently, Volkmann [41] extended this result to arbitrary finite sums of the form $\ell_{p_{1}} \oplus \ell_{p_{2}} \oplus \cdots \oplus \ell_{p_{m}}$ and $\ell_{p_{1}} \oplus \ell_{p_{2}} \oplus \cdots \oplus \ell_{p_{m-1}} \oplus c_{0}$, where $m \in\{2,3, \ldots\}$ and $1 \leqslant p_{1}<p_{2}<\cdots<p_{m}<\infty$.

Pietsch surveyed these results in his monograph [34] and provided further progress in some cases, observing in particular that there are infinitely many closed operator ideals on $L_{p}[0,1]$ for $p \in(1,2) \cup(2, \infty)$ and uncountably many on $C[0,1]$. Moreover, he formally asked whether there are infinitely many closed operator ideals on each of the spaces $L_{1}[0,1]$, $\ell_{p} \oplus \ell_{q}$ and $\ell_{p} \oplus c_{0}$ for $1 \leqslant p<q<\infty$. These questions have only recently been answered, all in the affirmative; we shall give further details below.

After a relatively quiet period, the study of closed operator ideals has gained new momentum since the turn of the millenium. Among the early progress were the first new full classifications of the closed operator ideals on a Banach space since Gramsch's and Luft's work, beginning with the space $\left(\bigoplus_{k \in \mathbb{N}} \ell_{2}^{k}\right)_{c_{0}}$ and its dual $\left(\bigoplus_{k \in \mathbb{N}} \ell_{2}^{k}\right)_{\ell_{1}}$ (see [26] and [27], respectively), and Daws' generalization [9] of Gramsch's and Luft's result to the other nonseparable sequence spaces $\ell_{p}(\Gamma)$ and $c_{0}(\Gamma)$ for an arbitrary uncountable index set $\Gamma$ and $p \in[1, \infty)$.

Subsequently, as a bi-product of Argyros and Haydon's spectacular solution [3] of the scalar-plus-compact problem, several new Banach spaces whose closed operator ideals can be classified have appeared, including [39], [30] and [21]. Another such classification is given in [20, Theorem 5.5], namely for the Banach space $C(K)$, where $K$ is Koszmider's Mrówka space constructed in [23] under the Continuum Hypothesis; see [24] for the construction of such a space within ZFC. We refer to [21, Remark 1.5] for a more detailed survey of the above results.

An important common feature of the spaces listed in the previous paragraph is that they are all "purpose-built", which is in sharp contrast to those we described before. We consider 
it a very interesting - and probably very difficult — challenge to find new examples of "classical" Banach spaces whose closed operator ideals can be classified (where "classical" can perhaps best be understood as "having been known, or at least accessible to Banach"). To substantiate this claim, we shall outline three further cases, where apparently "nice" Banach spaces have been shown to have very intricate lattices of closed operator ideals. Theorem 1.1 above in the case of the original Schreier space $X\left[\mathcal{S}_{1}\right]$ could arguably also be included in this list.

We begin with Figiel's reflexive Banach spaces which are not isomorphic to their cartesian squares [10]. These Banach spaces are manifestly "nice", being defined by entirely elementary means. Indeed, what Figiel showed is that for each strictly decreasing sequence $\left(p_{k}\right)$ in $(2, \infty)$ and each number $q \in\left(1, \inf p_{k}\right]$, there is a sequence $\left(n_{k}\right)$ in $\mathbb{N}$ such that the Banach space

$$
F=\left(\bigoplus_{k \in \mathbb{N}} \ell_{p_{k}}^{n_{k}}\right)_{\ell_{q}}
$$

satisfies: for each $m \in \mathbb{N}, F^{m+1}$ does not embed isomorphically into $F^{m}$. Now consider the Boolean algebra $\mathscr{P}(\mathbb{N}) /[\mathbb{N}]^{<\infty}$ which is the quotient of the power set of $\mathbb{N}$ modulo the ideal of finite subsets. Loy and the third-named author [25, Theorem 4.12] constructed an injective map from the ideal lattice of $\mathscr{P}(\mathbb{N}) /[\mathbb{N}]^{<\infty}$ into the closed ideal lattice of $\mathscr{B}(F)$ such that this map preserves the order in both directions. This implies in particular that $\mathscr{B}(F)$ has continuum many closed ideals. (The result stated in [25, Corollary 4.13] says "uncountably many", but the argument actually gives continuum many by using the existence of an almost disjoint family of subsets of $\mathbb{N}$ having the cardinality of the continuum.)

More recently, Schlumprecht and Zsák [37] launched the first successful attack on the above-mentioned questions of Pietsch by constructing a chain of continuum many closed operator ideals on $\ell_{p} \oplus \ell_{q}$ for $1<p<q<\infty$. The other cases of this question have subsequently also been resolved in [42], [38] and [12], so we now know that $\mathscr{B}\left(\ell_{p} \oplus \ell_{q}\right)$ and $\mathscr{B}\left(\ell_{p} \oplus c_{0}\right)$ contain uncountable chains of closed ideals whenever $1 \leqslant p<q<\infty$. (In fact, in all cases except $\mathscr{B}\left(\ell_{1} \oplus c_{0}\right)$, the chains have the cardinality of the continuum.)

Finally, in 2018 Johnson, Pisier and Schechtman [18] answered the remaining question of Pietsch by constructing a chain of continuum many closed operator ideals on $L_{1}[0,1]$. They also obtained similar conclusions for $C[0,1]$ (where previously only uncountably many closed operator ideals were known) and $L_{\infty}[0,1]$ (and therefore also for $\ell_{\infty}$ because $\ell_{\infty}$ and $L_{\infty}[0,1]$ are isomorphic as Banach spaces by a theorem of Pełczyński [32]), using a variant of their argument and duality, respectively.

Note: after the initial version of this paper was submitted, Johnson and Schechtman [19] have shown that $\mathscr{B}\left(L_{p}[0,1]\right)$ contains $2^{\mathfrak{c}}$ closed ideals for each $p \in(1, \infty) \backslash\{2\}$.

To conclude this survey, let us remark that the Tsirelson and Schreier spaces are not the first examples of separable Banach spaces having at least continuum many maximal operator ideals. Indeed, Mankiewicz [29] and Dales-Loy-Willis [8] have independently constructed separable Banach spaces $X$ such that $\mathscr{B}(X)$ admits a bounded, surjective algebra homomorphism $\varphi$ onto $\ell_{\infty}$, and therefore $\left\{\varphi^{-1}(\mathscr{M}): \mathscr{M}\right.$ is a maximal ideal of $\left.\ell_{\infty}\right\}$ is a family of cardinality $2^{\mathfrak{c}}$ of maximal ideals of $\mathscr{B}(X)$. 


\section{Preliminaries, including the Framework of The Proof of Theorem 1.1}

For a set $N, \mathscr{P}(N)$ denotes its power set, while $[N]$ and $[N]^{<\infty}$ are the sets of infinite and finite subsets of $N$, respectively. We write $|N|$ for the cardinality of $N$; the letter $\mathfrak{c}$ denotes the cardinality of the continuum.

For two non-empty subsets $M$ and $N$ of $\mathbb{N}$, we use the notation $M<N$ to indicate that $M$ is finite and $\max M<\min N$. By an interval in a subset $N$ of $\mathbb{R}$, we understand a set of form $J \cap N$, where $J$ is an interval of $\mathbb{R}$ in the usual sense. (Note that the interval $J$ may be open, closed or half-open.)

All normed spaces are over the same scalar field $\mathbb{K}$, which may be either the real or the complex numbers. The term "operator" means a bounded, linear map between normed spaces. We write $\mathscr{B}(X)$ for the Banach algebra of operators on a Banach space $X$. For $S \in \mathscr{B}(X),\langle S\rangle$ denotes the (algebraic, two-sided) ideal of $\mathscr{B}(X)$ generated by $S$, that is,

$$
\langle S\rangle=\left\{\sum_{j=1}^{k} U_{j} S V_{j}: k \in \mathbb{N}, U_{1}, \ldots, U_{k}, V_{1}, \ldots, V_{k} \in \mathscr{B}(X)\right\} .
$$

Since $\mathscr{B}(X)$ is a unital Banach algebra, the ideal $\langle S\rangle$ is proper if and only if its norm-closure $\overline{\langle S\rangle}$ is. The following related result [25, Lemma 4.9] is fundamental to our investigations.

Lemma 2.1. Let $\mathscr{I}$ be an ideal of a Banach algebra $\mathscr{A}$, and let $P \in \mathscr{A}$ be idempotent. Then $P \in \mathscr{I}$ if (and only if) $P \in \overline{\mathscr{I}}$.

Definition 2.2. Let $X$ and $Y$ be Banach spaces. An operator $S: X \rightarrow Y$ is:

- strictly singular if, for each $\varepsilon>0$, each infinite-dimensional subspace of $X$ contains a unit vector $x$ such that $\|S x\|<\varepsilon$; in other words, the restriction of $S$ to $W$ is not an isomorphic embedding for any infinite-dimensional subspace $W$ of $X$;

- inessential if $I_{X}+R S$ is a Fredholm operator (that is, has finite-dimensional kernel and cofinite-dimensional image) for each operator $R: Y \rightarrow X$, where $I_{X}$ denotes the identity operator on $X$.

We write $\mathscr{S}(X, Y)$ and $\mathscr{E}(X, Y)$ for the sets of strictly singular and inessential operators from $X$ to $Y$, respectively.

With these definitions, $\mathscr{S}$ and $\mathscr{E}$ define closed operator ideals in the sense of Pitsch, and $\mathscr{S}(X, Y) \subseteq \mathscr{E}(X, Y)$ for any Banach spaces $X$ and $Y$. As usual, we write $\mathscr{S}(X)$ and $\mathscr{E}(X)$ instead of $\mathscr{S}(X, X)$ and $\mathscr{E}(X, X)$. A projection $P \in \mathscr{B}(X)$ is inessential if and only if it has finite-dimensional image. Although we shall not require this result, let us mention that $\mathscr{E}(X)$ is equal to the pre-image under the quotient map of the Jacobson radical of the Calkin algebra $\mathscr{B}(X) / \mathscr{K}(X)$. This was indeed Kleinecke's original definition of the inessential operators on a single Banach space [22]; the definition given above, where the domain and codomain may differ, is due to Pietsch [34].

Pfaffenberger [33] has shown that $\mathscr{S}(X)=\mathscr{E}(X)$ whenever the Banach space $X$ is subprojective in the sense that each closed, infinite-dimensional subspace of $X$ contains a closed, infinite-dimensional subspace which is complemented in $X$. 
Let $X$ and $Y$ be Banach spaces. A basic sequence $\left(x_{j}\right)_{j \in \mathbb{N}}$ in $X$ dominates a basic sequence $\left(y_{j}\right)_{j \in \mathbb{N}}$ in $Y$ if there is a constant $C>0$ such that

$$
\left\|\sum_{j=1}^{k} \alpha_{j} y_{j}\right\| \leqslant C\left\|\sum_{j=1}^{k} \alpha_{j} x_{j}\right\| \quad\left(k \in \mathbb{N}, \alpha_{1}, \ldots, \alpha_{k} \in \mathbb{K}\right) .
$$

If we wish to record the value of the constant $C$, we say that $\left(x_{j}\right)_{j \in \mathbb{N}} C$-dominates $\left(y_{j}\right)_{j \in \mathbb{N}}$.

Let $X$ be a Banach space with an unconditional basis $\left(b_{j}\right)_{j \in \mathbb{N}}$. It is easy to see that the basis projections satisfy the identity

$$
P_{M \cup N}=P_{M}+P_{N}-P_{M \cap N} \quad(M, N \subseteq \mathbb{N}) .
$$

For a subset $M$ of $\mathbb{N}$, we write $X_{M}$ for the image of the basis projection $P_{M}$; that is,

$$
X_{M}=\overline{\operatorname{span}}\left\{b_{j}: j \in M\right\} .
$$

In the notation introduced above, the ideals of the form $\overline{\left\langle P_{M}\right\rangle}$ for some non-empty subset $M$ of $\mathbb{N}$ are precisely the spatial ideals of $\mathscr{B}(X)$. The ideal $\mathscr{K}(X)$ of compact operators is always spatial. More precisely, for $M \subseteq \mathbb{N}$, we have $\overline{\left\langle P_{M}\right\rangle}=\mathscr{K}(X)$ if and only if $M$ is nonempty and finite. The following lemma characterizes when one spatial ideal is contained in another.

Lemma 2.3. Let $X$ be a Banach space with an unconditional basis, and let $M$ and $N$ be subsets of $\mathbb{N}$. Then the following four conditions are equivalent:

(a) $P_{M} \in \overline{\left\langle P_{N}\right\rangle}$;

(b) $\left\langle P_{M}\right\rangle \subseteq\left\langle P_{N}\right\rangle$;

(c) $\left\langle P_{N}\right\rangle=\left\langle P_{M \cup N}\right\rangle$;

(d) $X_{M}$ is isomorphic to a complemented subspace of $X_{N}^{k}$ for some $k \in \mathbb{N}$.

Proof. Lemma 2.1 shows that (a) implies (b), which in turn implies (c) by (2.1). Clearly (c) implies (a), and finally the equivalence of (a) and (d) is a special case of [26, Lemma 4.7] (or the much earlier [35, Lemma 1] if we know that $X_{M} \cong X_{M} \oplus X_{M}$, which will be the case in our applications of this result.)

Corollary 2.4. Let $X$ be a Banach space with an unconditional basis, and let $N$ be a nonempty subset of $\mathbb{N}$. Then $\left\langle P_{N}\right\rangle=\left\langle P_{N \cup F}\right\rangle$ for each $F \in[\mathbb{N}]^{<\infty}$.

Proof. We have $P_{F} \in \mathscr{F}(X) \subseteq\left\langle P_{N}\right\rangle$ because the set $F$ is finite and the ideal $\mathscr{F}(X)$ of finite-rank operators is the smallest non-zero ideal of $\mathscr{B}(X)$. Hence the conclusion follows from Lemma 2.3.

Combining Lemma 2.3 with Pełczyński's Decomposition Method, we obtain the following conclusion.

Corollary 2.5. Let $X$ be a Banach space with an unconditional basis, and let $M$ and $N$ be subsets of $\mathbb{N}$ such that $X_{M}$ is isomorphic to $X_{M} \oplus X_{M}$ and $X_{N}$ is isomorphic to $X_{N} \oplus X_{N}$. Then $\left\langle P_{M}\right\rangle=\left\langle P_{N}\right\rangle$ if and only if $X_{M}$ and $X_{N}$ are isomorphic. 
Proposition 2.6. (Dichotomy for chains of spatial ideals) Let $X$ be a Banach space with an unconditional basis, and let $\Gamma$ be a chain of spatial ideals. Then either $\Gamma$ stabilizes, so that $\bar{\cup} \in \Gamma$, or the ideal $\bar{\cup}$ is not spatial.

Proof. The two statements are clearly mutually exclusive. Suppose that the second statement fails, so that $\overline{\bigcup \Gamma}=\overline{\left\langle P_{M}\right\rangle}$ for some non-empty subset $M$ of $\mathbb{N}$. We must show that the first statement is satisfied, that is, $\overline{\left\langle P_{M}\right\rangle} \in \Gamma$. Since a projection belongs to the closure of an ideal if and only if it belongs to the ideal itself by Lemma 2.1, we can find a non-empty subset $N$ of $\mathbb{N}$ such that $P_{M} \in\left\langle P_{N}\right\rangle$ and $\overline{\left\langle P_{N}\right\rangle} \in \Gamma$. Then

$$
\overline{\left\langle P_{M}\right\rangle} \subseteq \overline{\left\langle P_{N}\right\rangle} \subseteq \overline{\bigcup \Gamma}=\overline{\left\langle P_{M}\right\rangle}
$$

so we conclude that $\overline{\left\langle P_{M}\right\rangle}=\overline{\left\langle P_{N}\right\rangle} \in \Gamma$, as required.

We shall next state two technical lemmas which will form the core of the proof of Theorem 1.1. The set-up is as follows. Let $X$ be a Banach space with an unconditional basis, and suppose that $M \subseteq N$ are non-empty subsets of $\mathbb{N}$ such that $P_{N} \notin\left\langle P_{M}\right\rangle$. We note that $N$ is infinite because otherwise $P_{N} \in \mathscr{F}(X) \subseteq\left\langle P_{M}\right\rangle$. Further, we see that the set

$$
\Omega_{M, N}=\left\{\overline{\left\langle P_{L}\right\rangle}: M \subseteq L \subseteq N, P_{N} \notin \overline{\left\langle P_{L}\right\rangle}\right\}
$$

of spatial ideals of $\mathscr{B}(X)$ is partially ordered by inclusion, and also non-empty with a smallest element, namely $\overline{\left\langle P_{M}\right\rangle}$. We say that a chain $\Gamma$ in $\Omega_{M, N}$ is set-induced if there is an increasing sequence $\left(L_{j}\right)_{j \in \mathbb{N}}$ of subsets of $N$ such that $M \subseteq L_{1}$ and $\Gamma=\left\{\overline{\left\langle P_{L_{j}}\right\rangle}: j \in \mathbb{N}\right\}$.

Lemma 2.7. Let $X$ be a Banach space with an unconditional basis, and let $M \subseteq N$ be non-empty subsets of $\mathbb{N}$ such that $P_{N} \notin\left\langle P_{M}\right\rangle$.

I. A chain $\Gamma$ in $\Omega_{M, N}$ is set-induced if and only if either $\Gamma$ stabilizes and has order type $n$ for some $n \in \mathbb{N}$, or $\Gamma$ has order type $\omega$.

II. Suppose that the following two conditions are satisfied:

(II.i) each set-induced chain in $\Omega_{M, N}$ has an upper bound in $\Omega_{M, N}$;

(II.ii) $\Omega_{M, N}$ has no maximal elements.

Then each countable chain in $\Omega_{M, N}$ has an upper bound in $\Omega_{M, N}$, and there is a (necessarily uncountable) chain $\Gamma$ in $\Omega_{M, N}$ such that:

- $\Gamma$ has no upper bound in $\Omega_{M, N}$;

- each countable subchain of $\Gamma$ has an upper bound in $\Gamma$;

- the ideal $\cup \Gamma$ is closed, and it is not spatial.

III. Suppose that there is a map $\varphi: \mathscr{P}(\mathbb{N}) \rightarrow[N]$ which satisfies the following three conditions for each pair $D, E \in \mathscr{P}(\mathbb{N})$ :

(III.i) $M \subseteq \varphi(D)$;

(III.ii) $\varphi(D) \cup \varphi(E)=\varphi(D \cap E)$;

(III.iii) $P_{N} \in\left\langle P_{\varphi(D)}\right\rangle$ if and only if $D \in[\mathbb{N}]^{<\infty}$.

Then there is a family $\Delta \subseteq[N]$ of cardinality $\mathfrak{c}$ such that

$M \subseteq L \quad$ and $\quad \overline{\left\langle P_{M}\right\rangle} \varsubsetneqq \overline{\left\langle P_{L}\right\rangle} \varsubsetneqq \overline{\left\langle P_{N}\right\rangle}=\overline{\left\langle P_{L \cup L^{\prime}}\right\rangle} \quad\left(L, L^{\prime} \in \Delta, L \neq L^{\prime}\right)$. 
Proof. I. The forward implication is clear.

Conversely, let $\Gamma$ be a chain in $\Omega_{M, N}$ of order type $n \in \mathbb{N} \cup\{\omega\}$. In both cases we can express $\Gamma$ as $\Gamma=\left\{\overline{\left\langle P_{K_{j}}\right\rangle}: j \in \mathbb{N}\right\}$, where $M \subseteq K_{j} \subseteq N$ and $P_{N} \notin \overline{\left\langle P_{K_{j}}\right\rangle} \subseteq \overline{\left\langle P_{K_{j+1}}\right\rangle}$ for each $j \in \mathbb{N}$. Then, defining $L_{j}=\bigcup_{i=1}^{j} K_{i}$, we obtain an increasing sequence $\left(L_{j}\right)_{j \in \mathbb{N}}$ of subsets of $N$ such that $M \subseteq L_{1}$ and $\left\langle P_{K_{j}}\right\rangle=\left\langle P_{L_{j}}\right\rangle$ for each $j \in \mathbb{N}$ by Lemma 2.3. Consequently $\Gamma=\left\{\overline{\left\langle P_{L_{j}}\right\rangle}: j \in \mathbb{N}\right\}$ is set-induced.

II. Let $\Gamma$ be a countable chain in $\Omega_{M, N}$. If $\Gamma$ is finite, then it has a maximal element and thus an upper bound in $\Omega_{M, N}$. Otherwise we can choose a subset $\Upsilon$ of $\Gamma$ such that $\Upsilon$ has order type $\omega$ and every element of $\Gamma$ is contained in an element of $\Upsilon$. Then $\Upsilon$ is set-induced by I. Hence condition (II.i) implies that $\Upsilon$ has an upper bound in $\Omega_{M, N}$, and that upper bound is clearly also an upper bound for $\Gamma$.

If each chain in $\Omega_{M, N}$ had an upper bound in $\Omega_{M, N}$, then the Kuratowski-Zorn Lemma would imply that $\Omega_{M, N}$ contains a maximal element, contrary to condition (II.ii). Therefore $\Omega_{M, N}$ contains a chain $\Gamma$ without any upper bound in $\Omega_{M, N}$, and this chain $\Gamma$ must be uncountable by the result proved in the previous paragraph.

To establish the second bullet point, assume towards a contradiction that $\Gamma$ contains a countable subchain $\Upsilon$ which has no upper bound in $\Gamma$. As shown above, $\Upsilon$ has an upper bound $\mathscr{M} \in \Omega_{M, N}$. The assumption means that, for each $\mathscr{J} \in \Gamma$, we can find $\mathscr{L} \in \Upsilon$ such that $\mathscr{L} \nsubseteq \mathscr{J}$. Hence $\mathscr{J} \subseteq \mathscr{L}$ because $\Gamma$ is a chain, and therefore also $\mathscr{J} \subseteq \mathscr{M}$. This shows that $\mathscr{M}$ is an upper bound for $\Gamma$, which contradicts that $\mathscr{M} \in \Omega_{M, N}$.

To see that $\bigcup \Gamma$ is closed, suppose that $S \in \bar{U}$. We can then recursively construct a sequence $\left(S_{j}\right)$ of operators and an increasing sequence $\left(\mathscr{J}_{j}\right)$ of spatial ideals belonging to $\Gamma$ such that $S_{j} \in \mathscr{J}_{j}$ and $\left\|S-S_{j}\right\|<1 / j$ for each $j \in \mathbb{N}$. As we showed in the previous paragraph, the countable subchain $\left\{\mathscr{J}_{j}: j \in \mathbb{N}\right\}$ of $\Gamma$ has an upper bound $\mathscr{M} \in \Gamma$. Since $\mathscr{M}$ is closed and contains $S_{j}$ for each $j \in \mathbb{N}$, we conclude that $S \in \mathscr{M} \subseteq \bigcup \Gamma$, as required. Finally, since $\Gamma$ has no upper bound in $\Omega_{M, N}$, it cannot stabilize, so Proposition 2.6 implies that the ideal $\bigcup \Gamma$ is not spatial.

III. We begin by showing that $\overline{\left\langle P_{M}\right\rangle} \varsubsetneqq \overline{\left\langle P_{\varphi(D)}\right\rangle}$ whenever $D \subseteq \mathbb{N}$ is co-infinite. The inclusion follows from (III.i). To see that it is proper when $D$ is co-infinite, suppose that the two ideals are equal, so that $P_{\varphi(D)} \in\left\langle P_{M}\right\rangle$ by Lemma 2.1, and set $D^{c}=\mathbb{N} \backslash D$. Condition (III.i) implies that $P_{M} \in\left\langle P_{\varphi\left(D^{c}\right)}\right\rangle$, and hence also $P_{\varphi(D)} \in\left\langle P_{\varphi\left(D^{c}\right)}\right\rangle$. Combining this with Lemma 2.3 and (III.ii)-(III.iii), we deduce that

$$
\left\langle P_{\varphi\left(D^{c}\right)}\right\rangle=\left\langle P_{\varphi(D) \cup \varphi\left(D^{c}\right)}\right\rangle=\left\langle P_{\varphi(\emptyset)}\right\rangle \ni P_{N} .
$$

This shows that $D^{c}$ is finite by condition (III.iii), and the conclusion follows.

Now take a family $\mathscr{D} \subseteq[\mathbb{N}]$ of cardinality $\mathfrak{c}$ such that $\mathscr{D}$ is almost disjoint in the sense that $D \cap E$ is finite whenever $D, E \in \mathscr{D}$ are distinct, and set $\Delta=\varphi(\mathscr{D})$. Then each $D \in \mathscr{D}$ is infinite and co-infinite, so $\overline{\left\langle P_{M}\right\rangle} \varsubsetneqq \overline{\left\langle P_{\varphi(D)}\right\rangle} \varsubsetneqq \overline{\left\langle P_{N}\right\rangle}$ by the result proved in the previous paragraph and (III.iii). Suppose that $D, E \in \mathscr{D}$ are distinct. Then $D \cap E \in[\mathbb{N}]^{<\infty}$, so $P_{N} \in\left\langle P_{\varphi(D \cap E)}\right\rangle=\left\langle P_{\varphi(D) \cup \varphi(E)}\right\rangle$ by (III.iii) and (III.ii). This establishes the final equality in $(2.3)$, and it also implies that $\varphi(D) \neq \varphi(E)$, so $\varphi$ is injective, and thus $|\Delta|=|\mathscr{D}|=\mathfrak{c}$. 
Lemma 2.8. Let $X$ denote either Tsirelson's space $T$ or the Schreier space $X\left[\mathcal{S}_{n}\right]$ of order $n$ for some $n \in \mathbb{N}$.

(i) For each $N \in[\mathbb{N}]$, there is $M \in[N]$ such that $P_{N} \notin\left\langle P_{M}\right\rangle$, and consequently

$$
\overline{\left\langle P_{M}\right\rangle} \varsubsetneqq \overline{\left\langle P_{N}\right\rangle} \text {. }
$$

(ii) Suppose that $M \subseteq N$ are infinite subsets of $\mathbb{N}$ such that $P_{N} \notin\left\langle P_{M}\right\rangle$. Then:

- there is a map $\varphi: \mathscr{P}(\mathbb{N}) \rightarrow[N]$ which satisfies conditions (III.i)-(III.iii) in Lemma 2.7, and hence there is a family $\Delta \subseteq[N]$ of cardinality $\mathfrak{c}$ such that (2.3) is satisfied;

- each set-induced chain in $\Omega_{M, N}$ has an upper bound in $\Omega_{M, N}$.

The proof of Lemma 2.8 is non-trivial both for Tsirelson's space and for the Schreier spaces of finite order; we shall give these proofs in Sections 3 and 4, respectively. However, once the lemma is established, Theorem 1.1 follows fairly easily, as we shall now show.

Proof of Theorem 1.1, assuming Lemma 2.8. Applying Lemma 2.8(i)-(ii) in the particular case $N=\mathbb{N}$, we see that $\mathscr{B}(X)$ contains a non-trivial spatial ideal and that each proper spatial ideal has at least continuum many successors, so no such ideal is maximal. Another application of Lemma 2.8(i) shows that no non-trivial spatial ideal is minimal. This establishes Theorem 1.1(i).

To verify Theorem 1.1(ii), let $\mathscr{I} \varsubsetneqq \mathscr{J}$ be spatial ideals of $\mathscr{B}(X)$, and take non-empty subsets $K, N$ of $\mathbb{N}$ such that $\mathscr{I}=\overline{\left\langle P_{K}\right\rangle}$ and $\mathscr{J}=\overline{\left\langle P_{N}\right\rangle}$. By Lemma 2.3 , we may replace $N$ with $N \cup K$ to ensure that $K \subseteq N$. Moreover, we may suppose that $K$ is infinite. Indeed, Lemma 2.8(i) implies that $N$ contains an infinite subset $K^{\prime}$ such that $\overline{\left\langle P_{K^{\prime}}\right\rangle} \varsubsetneqq \mathscr{J}$, and if $K$ is finite, then $\overline{\left\langle P_{K}\right\rangle}=\mathscr{K}(X) \subseteq \overline{\left\langle P_{K^{\prime}}\right\rangle}$, so we may replace $K$ with $K^{\prime}$.

This enables us to apply Lemma 2.8 (ii) with $M=K$ to obtain a family $\Delta \subseteq[N]$ of cardinality $\mathfrak{c}$ such that

$$
K \subseteq L \quad \text { and } \quad \mathscr{I} \varsubsetneqq \overline{\left\langle P_{L}\right\rangle} \varsubsetneqq \mathscr{J}=\overline{\left\langle P_{L \cup L^{\prime}}\right\rangle} \quad\left(L, L^{\prime} \in \Delta, L \neq L^{\prime}\right),
$$

and each set-induced chain in $\Omega_{K, N}$ has an upper bound in $\Omega_{K, N}$. Take $L \in \Delta$. Then $P_{N} \notin\left\langle P_{L}\right\rangle$, and Lemma 2.8(ii) (this time applied with $M=L$ ) shows that the pair $L \subseteq N$ satisfies conditions (II.i)-(II.ii) in Lemma 2.7. Consequently $\Omega_{L, N}$ contains an uncountable chain $\Gamma_{L}$ such that $\bigcup \Gamma_{L}$ is a closed ideal that is not spatial. It follows that the first two bullet points in Theorem 1.1(ii) are satisfied. To verify the third, suppose that $\mathscr{L} \in \Gamma_{L}$ and $\mathscr{M} \in \Gamma_{L^{\prime}}$, where $L, L^{\prime} \in \Delta$ are distinct. Then $P_{L} \in \mathscr{L}$ and $P_{L^{\prime}} \in \mathscr{M}$, and therefore (2.1) shows that $P_{L \cup L^{\prime}} \in \mathscr{L}+\mathscr{M}$. Hence the conclusion follows from the fact that $\overline{\left\langle P_{L \cup L^{\prime}}\right\rangle}=\mathscr{J}$ by $(2.4)$.

(iii). Applying clause (ii) in the case where $\mathscr{J}=\mathscr{B}(X)$ and $\mathscr{I}$ is any proper spatial ideal, we deduce that there is a family $\Xi$ of proper spatial ideals of $\mathscr{B}(X)$ such that $\Xi$ has cardinality $\mathfrak{c}$ and

$$
\overline{\mathscr{L}+\mathscr{M}}=\mathscr{B}(X) \quad(\mathscr{L}, \mathscr{M} \in \Xi, \mathscr{L} \neq \mathscr{M})
$$


Each of the ideals in $\Xi$ is contained in a maximal ideal of $\mathscr{B}(X)$, and (2.5) implies that these maximal ideals are all distinct, so $\mathscr{B}(X)$ contains at least continuum many maximal ideals.

\section{TSIRELSON'S SPACE}

Following Figiel and Johnson [11], we use the term Tsirelson's space for the dual of the reflexive Banach space that Tsirelson [40] originally constructed with the property that it does not contain any of the classical sequence spaces $c_{0}$ and $\ell_{p}$ for $1 \leqslant p<\infty$, and we denote it by $T$. This convention makes no difference from the point of view of ideal lattices because $T$ is reflexive, so the adjoint map $S \mapsto S^{*}, \mathscr{B}(T) \rightarrow \mathscr{B}\left(T^{*}\right)$, is an isometric, linear bijection which is anti-multiplicative in the sense that $(R S)^{*}=S^{*} R^{*}$ for $R, S \in \mathscr{B}(T)$, and therefore it induces a lattice isomorphism between the closed ideal lattices of $\mathscr{B}(T)$ and $\mathscr{B}\left(T^{*}\right)$.

We refer to Casazza and Shura's monograph [7] for details about Tsirelson's space, including its formal definition. In line with their notation, we write $\left(t_{j}\right)_{j \in \mathbb{N}}$ for the unit vector basis, which is a normalized, 1-unconditional basis for $T$. Recall from (2.2) that $T_{M}$ denotes the closed linear span of $\left\{t_{j}: j \in M\right\}$ in $T$ for a subset $M$ of $\mathbb{N}$. Using this notation, we have the following fundamental result [7, Corollary VII.b.3].

Theorem 3.1. Let $M, N \in[\mathbb{N}]$. Then $T_{M}$ is isomorphic to $T_{N}$ if and only if $\left(t_{j}\right)_{j \in M}$ is equivalent to $\left(t_{j}\right)_{j \in N}$.

The usefulness of this result relies on being able to determine when two subsequences of the basis $\left(t_{j}\right)$ are equivalent. Fortunately, Casazza, Johnson and Tzafriri [6] have identified an index which does exactly that. To define it, we require some notation. First, for $M=\left\{m_{1}<m_{2}<\cdots\right\} \in[\mathbb{N}]$ and $J \in[\mathbb{N}]^{<\infty}$, let $\sigma(M, J)$ denote the norm of the formal identity operator from the linear span of $\left(t_{m_{j}}\right)_{j \in J}$ to $\ell_{1}(J)$, that is,

$$
\sigma(M, J)=\sup \left\{\sum_{j \in J} \alpha_{j}: \alpha_{j} \in[0,1],\left\|\sum_{j \in J} \alpha_{j} t_{m_{j}}\right\|_{T} \leqslant 1\right\},
$$

with the convention that $\sigma(M, \emptyset)=0$. Second, suppose that $N=\left\{n_{1}<n_{2}<\cdots\right\} \in[\mathbb{N}$, and set $m_{0}=n_{0}=0$. Then Casazza, Johnson and Tzafriri have shown that $\left(t_{j}\right)_{j \in M}$ is equivalent to $\left(t_{j}\right)_{j \in N}$ if and only if

$$
\sup \left\{\sigma\left(M, M \cap\left(n_{j-1}, n_{j}\right]\right), \sigma\left(N, N \cap\left(m_{j-1}, m_{j}\right]\right): j \in \mathbb{N}\right\}<\infty
$$

(see [6, the remark following Theorem 10]). This result simplifies considerably in the special case where $M \subseteq N$, which will suffice for our purposes. We incorporate it in the following omnibus characterization of equality of spatial ideals of $\mathscr{B}(T)$, which will be our key tool in the proof of Lemma 2.8 for Tsirelson's space.

Corollary 3.2. The following conditions are equivalent for each pair $M \subseteq N$ of infinite subsets of $\mathbb{N}$ :

(a) $P_{N} \in \overline{\left\langle P_{M}\right\rangle}$; 
(b) $\left\langle P_{M}\right\rangle=\left\langle P_{N}\right\rangle$;

(c) $T_{N}$ is isomorphic to a complemented subspace of $T_{M}$;

(d) $T_{N}$ is isomorphic to $T_{M}$;

(e) $\left(t_{j}\right)_{j \in M}$ is equivalent to $\left(t_{j}\right)_{j \in N}$;

(f) there is a constant $C \geqslant 1$ such that $\sigma(N, J) \leqslant C$ for each interval $J$ in $N$ with $J \cap M=\emptyset$.

As it will be used repeatedly in the remainder of this section, let us spell out that the conditions on the set $J$ in clause (f) above mean that $J=N \cap[a, b]$ for some numbers $a, b$ satisfying $m_{j-1}<a \leqslant b<m_{j}$ for some $j \in \mathbb{N}$, where $m_{0}=0$ and $M=\left\{m_{1}<m_{2}<\cdots\right\}$ is the increasing enumeration of $M$, as above.

Proof. The assumption that $M \subseteq N$ means that $P_{M}=P_{M} P_{N} \in\left\langle P_{N}\right\rangle$, and hence conditions (a)-(d) are equivalent by Lemma 2.3 and Corollary 2.5 because $T_{L} \cong T_{L} \oplus T_{L}$ for each $L \in[\mathbb{N}]$, as explained in [7, the paragraph following Proposition I.12]. Theorem 3.1 shows that conditions (d) and (e) are equivalent.

Finally, conditions (e) and (f) are equivalent by the result of Casazza, Johnson and Tzafriri stated above. Indeed, suppose that (e) is satisfied, and let $C \in[1, \infty)$ be the supremum given by (3.1). Since each interval $J$ in $N$ with $J \cap M=\emptyset$ is contained in $N \cap\left(m_{j-1}, m_{j}\right)$ for some $j \in \mathbb{N}$, we have $\sigma(N, J) \leqslant \sigma\left(N, N \cap\left(m_{j-1}, m_{j}\right]\right) \leqslant C$.

Conversely, suppose that $C$ is a constant such that (f) is satisfied, and take $j \in \mathbb{N}$. Since $M \subseteq N$, the set $M \cap\left(n_{j-1}, n_{j}\right]$ is either empty or a singleton, so $\sigma\left(M, M \cap\left(n_{j-1}, n_{j}\right]\right) \leqslant 1$. Moreover, the subadditivity of the operator norm implies that

$$
\sigma\left(N, N \cap\left(m_{j-1}, m_{j}\right]\right) \leqslant \sigma\left(N, N \cap\left(m_{j-1}, m_{j}\right)\right)+1 \leqslant C+1,
$$

and hence the supremum in (3.1) is at most $C+1$.

Proof of Lemma 2.8 for $X=T$. (i). Let $N=\left\{n_{1}<n_{2}<\cdots\right\} \in[\mathbb{N}]$. For each $k \in \mathbb{N}$, we can find $m>k$ such that $\sigma(N, N \cap(k, m))>k$ because otherwise the basic sequence $\left(t_{n_{j}}\right)_{j \in N \cap(k, \infty)}$ would $k$-dominate, and hence be equivalent to, the unit vector basis of $\ell_{1}$ for some $k \in \mathbb{N}$. Using this observation, we can recursively construct a strictly increasing sequence $\left(m_{j}\right)$ in $N$ such that $\sigma\left(N, N \cap\left(m_{j-1}, m_{j}\right)\right)>m_{j-1}$ for each $j \in \mathbb{N}$, where $m_{0}=0$. Now Corollary 3.2 shows that the subset $M=\left\{m_{1}<m_{2}<\cdots\right\}$ of $N$ has the desired property.

(ii). Let $M \subseteq N$ be infinite subsets of $\mathbb{N}$ such that $P_{N} \notin\left\langle P_{M}\right\rangle$. By Corollary 3.2, we can recursively choose intervals $J_{1}<J_{2}<\cdots$ in $N$ such that $J_{k} \cap M=\emptyset$ and $\sigma\left(N, J_{k}\right) \geqslant k$ for each $k \in \mathbb{N}$. We shall show that the map $\varphi: \mathscr{P}(\mathbb{N}) \rightarrow[N]$ defined by

$$
\varphi(D)=N \backslash \bigcup_{j \in D} J_{j}
$$

satisfies conditions (III.i)-(III.iii) in Lemma 2.7. The first two of these conditions are immediate. To verify the third, suppose that $D \subseteq \mathbb{N}$ is finite. Then the set $\bigcup_{j \in D} J_{j}$ is also finite, and therefore $P_{N} \in\left\langle P_{\varphi(D)}\right\rangle$ by Corollary 2.4. Conversely, suppose that $D \subseteq \mathbb{N}$ is infinite. For each $k \in D, J_{k}$ is an interval in $N$ such that $J_{k} \cap \varphi(D)=\emptyset$ and $\sigma\left(N, J_{k}\right) \geqslant k$, 
so as $D$ is unbounded, Corollary 3.2 implies that $P_{N} \notin\left\langle P_{\varphi(D)}\right\rangle$. This establishes (III.iii) and hence completes the proof of the first bullet point.

To verify the second, let $\Gamma$ be a set-induced chain in $\Omega_{M, N}$, say $\Gamma=\left\{\overline{\left\langle P_{L_{j}}\right\rangle}: j \in \mathbb{N}\right\}$, where $M \subseteq L_{j} \subseteq L_{j+1} \subseteq N$ and $P_{N} \notin \overline{\left\langle P_{L_{j}}\right\rangle}$ for each $j \in \mathbb{N}$. By Corollary 3.2, we may recursively construct intervals $J_{1}<J_{2}<\cdots$ in $N$ such that $\sigma\left(N, J_{j}\right) \geqslant j$ and $J_{j} \cap L_{j}=\emptyset$ for each $j \in \mathbb{N}$. Set $K=N \backslash \bigcup_{j \in \mathbb{N}} J_{j}$. Then $M \subseteq K \subseteq N$, and $P_{N} \notin \overline{\left\langle P_{K}\right\rangle}$ by Corollary 3.2 because $J_{j}$ is an interval in $N$ with $K \cap J_{j}=\emptyset$ and $\sigma\left(N, J_{j}\right) \geqslant j$ for each $j \in \mathbb{N}$. Hence $\overline{\left\langle P_{K}\right\rangle}$ belongs to $\Omega_{M, N}$. Moreover, for each $k \in \mathbb{N}$ and $j \geqslant k$, we have $J_{j} \cap L_{k} \subseteq J_{j} \cap L_{j}=\emptyset$, so $L_{k} \subseteq K \cup \bigcup_{j<k} J_{j}$. Since $\bigcup_{j<k} J_{j}$ is finite, Corollary 2.4 implies that $P_{L_{k}} \in\left\langle P_{K}\right\rangle$, and therefore $\overline{\left\langle P_{K}\right\rangle}$ is an upper bound for $\Gamma$.

We require the following result, which is [7, Proposition II.7], to prove Theorem 1.2(i).

Theorem 3.3. Every closed, infinite-dimensional subspace of $T$ contains a closed subspace which is complemented in $T$ and isomorphic to $T_{N}$ for some $N \in[\mathbb{N}]$.

Proof of Theorem 1.2(i). A standard perturbation argument shows that $\mathscr{K}(T)=\mathscr{S}(T)$, as remarked in [2, p. 1173], for instance. As observed in [16, Proposition 2.4(5)], Theorem 3.3 implies that $T$ is subprojective, and therefore $\mathscr{S}(T)=\mathscr{E}(T)$ by the result [33] of Pfaffenberger mentioned on page 5 .

The inclusion

$$
\mathscr{K}(T) \subseteq \bigcap\{\mathscr{I}: \mathscr{I} \text { is a non-trivial spatial ideal of } \mathscr{B}(T)\}
$$

is clear. Conversely, suppose that $S \in \mathscr{B}(T) \backslash \mathscr{K}(T)$. Then, as explained in the first paragraph, $S$ is not strictly singular. Take a closed, infinite-dimensional subspace $W$ of $T$ such that the restriction of $S$ to $W$ is an isomorphism onto its image $S(W)$. Then $S(W)$ is a closed, infinite-dimensional subspace of $T$, so Theorem 3.3 implies that $S(W)$ contains a closed subspace $Z$ which is complemented in $T$ and isomorphic to $T_{N}$ for some $N \in[\mathbb{N}]$. Let $Q: T \rightarrow Z$ be a projection, and let $U: Z \rightarrow T_{N}$ be an isomorphism. We observe that the restriction of $S$ to the subspace $Y=S^{-1}(Z) \cap W$ is an isomorphism onto $Z$; denote it by $\widetilde{S}$. Then we have a commutative diagram

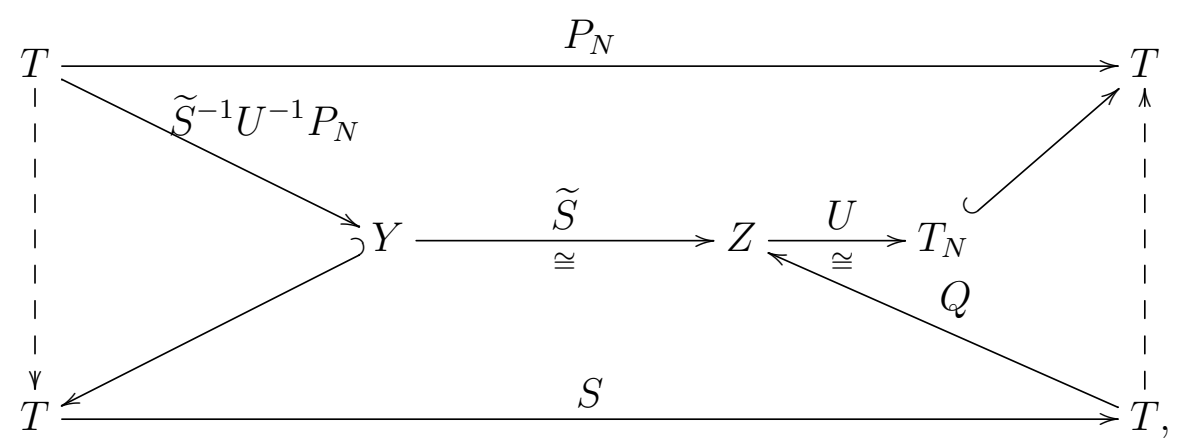

where the two unlabelled solid arrows are the set-theoretic inclusions. This diagram shows that $P_{N}$ factors through $S$, and therefore $P_{N} \in\langle S\rangle$. By Lemma 2.8(i), we can find $M \in[N]$ such that $P_{N} \notin \overline{\left\langle P_{M}\right\rangle}$. Consequently $S \notin \overline{\left\langle P_{M}\right\rangle}$, so $S$ does not belong to the right-hand side of $(3.2)$. 


\section{The SChreIER SPACES OF FINITE ORDER}

The aim of this section is to establish Lemma 2.8 and Theorem 1.2(ii) for the Schreier space $X\left[\mathcal{S}_{n}\right]$ of order $n \in \mathbb{N}$ associated with the Schreier family $\mathcal{S}_{n}$, originally defined by Alspach and Argyros [1]. Their precise definition is as follows.

Definition 4.1. Let

$$
\mathcal{S}_{0}=\{\{k\}: k \in \mathbb{N}\} \cup\{\emptyset\},
$$

and for $n \in \mathbb{N}_{0}$, recursively define

$$
\mathcal{S}_{n+1}=\left\{\bigcup_{i=1}^{k} E_{i}: k \in \mathbb{N}, E_{1}, \ldots, E_{k} \in \mathcal{S}_{n} \backslash\{\emptyset\}, k \leqslant \min E_{1}, E_{1}<E_{2}<\cdots<E_{k}\right\} \cup\{\emptyset\} .
$$

For $n \in \mathbb{N}_{0}$, the Schreier space of order $n$ is the completion of $c_{00}$ with respect to the norm

$$
\|x\|=\sup \left\{\sum_{j \in E}\left|\alpha_{j}\right|: E \in \mathcal{S}_{n} \backslash\{\emptyset\}\right\} \quad\left(x=\left(\alpha_{j}\right)_{j \in \mathbb{N}} \in c_{00}\right) .
$$

We denote this Banach space by $X\left[\mathcal{S}_{n}\right]$.

Of course, the Schreier space of order 0 is simply $c_{0}$. For a fixed order $n \in \mathbb{N}_{0}$, we write $\left(e_{j}\right)_{j \in \mathbb{N}}$ for the unit vector basis for $X\left[\mathcal{S}_{n}\right]$. Alspach and Argyros [1] have shown that this basis is 1-unconditional and shrinking.

Note: the original definition of Alspach and Argyros of the Schreier family $\mathcal{S}_{n}$ has an extension to the case where $n$ is a countably infinite ordinal. We have stated it for finite $n$ only because we have been unable to extend our results beyond that case.

Each subset of a set in $\mathcal{S}_{n}$ is clearly also in $\mathcal{S}_{n}$. Another elementary and often useful property of the Schreier family $\mathcal{S}_{n}$ is that it is spreading in the following sense. Let $J=\left\{j_{1}<j_{2}<\cdots<j_{m}\right\}$ and $K=\left\{k_{1}<k_{2}<\cdots<k_{m}\right\}$ be finite subsets of $\mathbb{N}$, and suppose that $K$ is a spread of $J$; that is, $j_{i} \leqslant k_{i}$ for each $i \in\{1, \ldots, m\}$. Then $J \in \mathcal{S}_{n}$ implies that $K \in \mathcal{S}_{n}$.

We shall require several results and definitions from the paper [14] of Gasparis and Leung that we shall now review. However, the starting point is a result of Gasparis alone [13, Corollary 3.2], which states that, for each $n \in \mathbb{N}_{0}$ and $M \in[\mathbb{N}]$, there is a unique sequence $\left(F_{j}^{n}(M)\right)_{j \in \mathbb{N}}$ of finite subsets of $M$ such that:

(1) $M=\bigcup_{j \in \mathbb{N}} F_{j}^{n}(M)$;

(2) $F_{j}^{n}(M)$ is a maximal $\mathcal{S}_{n}$-set for each $j \in \mathbb{N}$ in the sense that $E=F_{j}^{n}(M)$ is the only set $E \in \mathcal{S}_{n}$ such that $F_{j}^{n}(M) \subseteq E$;

(3) the sets $F_{j}^{n}(M)$ are successive in the sense that $F_{j}^{n}(M)<F_{j+1}^{n}(M)$ for each $j \in \mathbb{N}$. Gasparis and Leung [14, Definition 3.1] used this result to define the following numerical index for a set $J \in[\mathbb{N}]^{<\infty}$ :

$$
\tau_{n}(J)=\max \left\{k \in \mathbb{N}: J \cap F_{k}^{n}\left(J_{\text {tail }}\right) \neq \emptyset\right\},
$$

where we have introduced the notation $J_{\text {tail }}=J \cup\{j \in \mathbb{N}: j>\max J\}$ in an attempt to make the expression a little easier to comprehend. Roughly speaking, $\tau_{n}(J)$ counts how 
many successive maximal $\mathcal{S}_{n}$-sets (almost) fit inside $J$. The following remark collects three easy observations concerning this index.

Remark 4.2. Let $n \in \mathbb{N}_{0}$ and $J \in[\mathbb{N}]^{<\infty}$. Then:

(i) $\tau_{n}(J) \geqslant 1$ if and only if $J \neq \emptyset$.

(ii) For $k \in \mathbb{N}, \tau_{n}(J)=k$ if and only if there are successive $\mathcal{S}_{n^{-}}$sets $E_{1}<E_{2}<\cdots<E_{k}$ such that $J=\bigcup_{i=1}^{k} E_{i}$ and $E_{1}, \ldots, E_{k-1}$ are maximal $\mathcal{S}_{n}$-sets. (Note that the final $\mathcal{S}_{n}$-set $E_{k}$ need not be maximal.)

(iii) Suppose that $J$ is non-empty. Then

$$
\tau_{n}(J)=\min \left\{k \in \mathbb{N}: J \subseteq \bigcup_{i=1}^{k} E_{i}, \text { where } E_{1}, \ldots, E_{k} \in \mathcal{S}_{n} \text { and } E_{1}<E_{2}<\cdots<E_{k}\right\} .
$$

As Gasparis and Leung observed [14, Lemma 3.2(2)], the latter formula implies that $\tau_{n}$ is subadditive in the sense that

$$
\tau_{n}(J \cup K) \leqslant \tau_{n}(J)+\tau_{n}(K)
$$

whenever $J, K \in[\mathbb{N}]^{<\infty}$ are successive.

Gasparis and Leung used the index $\tau_{n}$ to define another index $d_{n}$, which can be viewed as a (not necessarily symmetric) way of measuring the distance from one infinite subset of $\mathbb{N}$ to another in terms of the Schreier family $\mathcal{S}_{n}$. To help state the definition of $d_{n}$ clearly and compactly, we introduce the following piece of notation, which was not used by Gasparis and Leung: let $J$ and $K$ be (finite or infinite) subsets of $\mathbb{N}$ such that $\sup J \leqslant|K|$ (so that in particular $K$ is infinite whenever $J$ is infinite), and enumerate $K$ in increasing order: $K=\left\{k_{1}<k_{2}<\cdots\right\}$. Then we set

$$
K(J)=\left\{k_{j}: j \in J\right\} .
$$

Definition 4.3 (Gasparis and Leung [14, Definition 3.3]). Let $n \in \mathbb{N}_{0}$ and $M, N \in[\mathbb{N}]$. The $n^{\text {th }}$ Gasparis-Leung index of $M$ with respect to $N$ is given by

$$
d_{n}(M, N)=\sup \left\{\tau_{n}(M(J)): J \in[\mathbb{N}]^{<\infty}, N(J) \in \mathcal{S}_{n}\right\} .
$$

We note that $d_{n}(M, N)=1$ whenever $M \subseteq N$ because in this case $M(J)$ is a spread of $N(J)$ for each $J \in[\mathbb{N}]^{<\infty}$.

The significance of the Gasparis-Leung index $d_{n}$ is due to the following result (see [14, Corollary 1.2(1) and Lemma 3.4, including its proof]), which together with the immediate consequence that we record in Corollary 4.5 will be a key tool for us. Recall that the notation $X_{N}$ was introduced in (2.2).

Theorem 4.4 (Gasparis and Leung). Let $X=X\left[\mathcal{S}_{n}\right]$ for some $n \in \mathbb{N}$, and let $M, N \in[\mathbb{N}]$.

(i) Suppose that $X_{M}$ is isomorphic to a subspace of $X_{N}$. Then the basic sequence $\left(e_{j}\right)_{j \in M}$ dominates $\left(e_{j}\right)_{j \in N}$.

(ii) The basic sequence $\left(e_{j}\right)_{j \in M}$ dominates $\left(e_{j}\right)_{j \in N}$ if and only if $d_{n}(M, N)$ is finite. Moreover, when $d_{n}(M, N)$ is finite, $\left(e_{j}\right)_{j \in M} d_{n}(M, N)$-dominates $\left(e_{j}\right)_{j \in N}$. 
Corollary 4.5. Let $X=X\left[\mathcal{S}_{n}\right]$ for some $n \in \mathbb{N}$, and let $M, N \in[\mathbb{N}]$. Then the following three conditions are equivalent:

(a) the subspaces $X_{M}$ and $X_{N}$ are isomorphic;

(b) $X_{M}$ is isomorphic to a subspace of $X_{N}$, and $X_{N}$ is isomorphic to a subspace of $X_{M}$;

(c) $d_{n}(M, N)$ and $d_{n}(N, M)$ are both finite.

Our first application of these results is to establish the following proposition.

Proposition 4.6. Let $X=X\left[\mathcal{S}_{n}\right]$ for some $n \in \mathbb{N}$. Then $X_{M}$ is isomorphic to $X_{M} \oplus X_{M}$ for each $M \in[\mathbb{N}]$.

For clarity of presentation, we have split the proof into a series of lemmas.

Lemma 4.7. Let $n \in \mathbb{N}_{0}$, and let $\sigma: \mathbb{N} \rightarrow \mathbb{N}$ be a strictly increasing map. Then the left shift

$$
L_{\sigma}: \quad \sum_{j} \alpha_{j} e_{j} \mapsto \sum_{j} \alpha_{\sigma(j)} e_{j}, \quad c_{00} \rightarrow c_{00},
$$

extends to an operator of norm one on $X\left[\mathcal{S}_{n}\right]$.

Proof. For $x=\left(\alpha_{j}\right)_{j \in \mathbb{N}} \in c_{00}$, choose $E \in \mathcal{S}_{n} \backslash\{\emptyset\}$ such that $\left\|L_{\sigma} x\right\|=\sum_{j \in E}\left|\alpha_{\sigma(j)}\right|$. The set $\sigma(E)$ is a spread of $E$ because $\sigma$ is strictly increasing. Hence $\sigma(E) \in \mathcal{S}_{n}$, and therefore

$$
\|x\| \geqslant \sum_{k \in \sigma(E)}\left|\alpha_{k}\right|=\sum_{j \in E}\left|\alpha_{\sigma(j)}\right|=\left\|L_{\sigma} x\right\| .
$$

Lemma 4.8. Let $n \in \mathbb{N}$, and let $E<F$ be successive maximal $\mathcal{S}_{n}$-sets. Then:

(i) $|E \cup F| \geqslant 3 \min E$.

(ii) Suppose that $\min E \geqslant 2$. Then $2(E \cup F) \notin \mathcal{S}_{n}$.

Proof. (i). The maximality of $E$ and $F$ means that $|E| \geqslant \min E$ and

$$
|F| \geqslant \min F \geqslant \max E+1 \geqslant \min E+|E| \geqslant 2 \min E,
$$

and hence we have $|E \cup F|=|E|+|F| \geqslant 3 \min E$.

(ii). We proceed by induction on $n$.

The result follows easily from (i) for $n=1$ because in this case we have

$$
|2(E \cup F)|=|E \cup F| \geqslant 3 \min E>2 \min E=\min 2(E \cup F),
$$

so that $2(E \cup F) \notin \mathcal{S}_{1}$.

Now assume inductively that the result holds for some $n \in \mathbb{N}$. To prove it for $n+1$, let $E<F$ be maximal $\mathcal{S}_{n+1}$-sets with $k:=\min E \geqslant 2$, and set $\ell=\min F$. Then we can find maximal $\mathcal{S}_{n}$-sets $E_{1}<\cdots<E_{k}<E_{k+1}<\cdots<E_{k+\ell}$ such that $E=\bigcup_{i=1}^{k} E_{i}$ and $F=\bigcup_{i=1}^{\ell} E_{k+i}$. Applying (i) to the maximal $\mathcal{S}_{n}$-sets $E_{1}<E_{2}$, both of which are contained in $E$, we obtain $|E| \geqslant\left|E_{1} \cup E_{2}\right| \geqslant 3 \min E_{1}=3 k$, and therefore

$$
4 k \leqslant \min E+|E| \leqslant \max E+1 \leqslant \ell .
$$


In particular $4 k \leqslant k+\ell$, so that

$$
\bigcup_{i=1}^{2 k} 2\left(E_{2 i-1} \cup E_{2 i}\right) \subseteq 2(E \cup F),
$$

where $2\left(E_{1} \cup E_{2}\right), 2\left(E_{3} \cup E_{4}\right), \ldots, 2\left(E_{4 k-1} \cup E_{4 k}\right)$ are successive sets which do not belong to $\mathcal{S}_{n}$ by the induction hypothesis. Hence, if $2(E \cup F)$ is written as the union of $m$ successive $\mathcal{S}_{n}$-sets for some $m \in \mathbb{N}$, then we must have $m>2 k=\min 2(E \cup F)$. This shows that $2(E \cup F) \notin \mathcal{S}_{n+1}$, and hence the induction continues.

Corollary 4.9. For each $n \in \mathbb{N}, d_{n}(\mathbb{N}, 2 \mathbb{N}) \leqslant 3$, and hence the right shift given by

$$
R: \quad \sum_{j} \alpha_{j} e_{j} \mapsto \sum_{j} \alpha_{j} e_{2 j}, \quad c_{00} \rightarrow c_{00},
$$

extends to an operator of norm at most 3 on $X\left[\mathcal{S}_{n}\right]$.

Proof. Using (4.2) and Definition 4.3, we see that $d_{n}(\mathbb{N}, 2 \mathbb{N}) \leqslant 3$ if and only if $\tau_{n}(J) \leqslant 3$ for each $J \in[\mathbb{N}]^{<\infty}$ with $2 J \in \mathcal{S}_{n}$. By contraposition, the latter statement is equivalent to the statement that $2 J \notin \mathcal{S}_{n}$ for each $J \in[\mathbb{N}]^{<\infty}$ with $\tau_{n}(J) \geqslant 4$. To verify this, suppose that $J \in[\mathbb{N}]^{<\infty}$ with $\tau_{n}(J) \geqslant 4$. Since $\{1\}$ is a maximal $\mathcal{S}_{n}$-set, we have $\tau_{n}(J \backslash\{1\}) \geqslant 3$, so by Remark 4.2(ii), there are maximal $\mathcal{S}_{n}$-sets $E<F$ such that $E \cup F \subseteq J \backslash\{1\}$. Lemma 4.8(ii) then implies that $2(E \cup F)$ does not belong to $\mathcal{S}_{n}$, and the same is therefore true for its superset $2 J$, as desired.

To establish that the right shift $R$ given by (4.3) is bounded by 3 , we simply combine the inequality $d_{n}(\mathbb{N}, 2 \mathbb{N}) \leqslant 3$ with Theorem $4.4($ ii) to deduce that

$$
\left\|\sum_{j=1}^{k} \alpha_{j} e_{2 j}\right\| \leqslant 3\left\|\sum_{j=1}^{k} \alpha_{j} e_{j}\right\| \quad\left(k \in \mathbb{N}, \alpha_{1}, \ldots, \alpha_{k} \in \mathbb{K}\right) .
$$

The following example shows that we cannot in general lower the upper bound 3 on the quantity $d_{n}(\mathbb{N}, 2 \mathbb{N})$ in the above proof. It also shows that it is possible to have $\|R\|>2$.

Example 4.10. Let $n=2$, and consider the set $J=\{1,2, \ldots, 8\}$. We see that $\tau_{2}(J)=3$ because $J=\{1\} \cup\{2,3,4,5,6,7\} \cup\{8\}$, where the first two sets on the right-hand side are maximal $\mathcal{S}_{2}$-sets. However, $2 J$ belongs to $\mathcal{S}_{2}$ because it is the union of the two $\mathcal{S}_{1}$-sets $\{2,4\}$ and $\{6,8,10,12,14,16\}$. Hence $d_{2}(\mathbb{N}, 2 \mathbb{N}) \geqslant 3$.

Set $x=e_{1}+\frac{1}{6}\left(e_{2}+e_{3}+\cdots+e_{8}\right) \in X\left[\mathcal{S}_{2}\right]$. Then the above reasoning shows that $\|x\|=1$ (attained at $\{1\}$ and at any 6 -element subset of $\{2,3, \ldots, 8\}$ ), but $\|R x\|=1+\frac{7}{6}>2$ because its support belongs to $\mathcal{S}_{2}$.

Lemma 4.11. Let $n \in \mathbb{N}$ and $M \in[\mathbb{N}]$, and set $M^{\prime}=(2 M-1) \cup(2 M) \in[\mathbb{N}]$. Then

$$
d_{n}\left(M, M^{\prime}\right) \leqslant 3 \quad \text { and } \quad d_{n}\left(M^{\prime}, M\right) \leqslant 2,
$$

and hence $X_{M} \cong X_{M^{\prime}}$, where $X=X\left[\mathcal{S}_{n}\right]$. 
Proof. Write $M=\left\{m_{1}<m_{2}<\cdots\right\}$ and $M^{\prime}=\left\{m_{1}^{\prime}<m_{2}^{\prime}<\cdots\right\}$, where $m_{2 j-1}^{\prime}=2 m_{j}-1$ and $m_{2 j}^{\prime}=2 m_{j}$ for each $j \in \mathbb{N}$.

To prove the first inequality in (4.4), suppose that $J \in[\mathbb{N}]^{<\infty}$ with $M^{\prime}(J) \in \mathcal{S}_{n}$. The definitions above imply that $m_{j}^{\prime} \leqslant 2 m_{j}$ for each $j \in \mathbb{N}$, so that $2 M(J)$ is a spread of $M^{\prime}(J)$ and therefore $2 M(J) \in \mathcal{S}_{n}$. Hence $\tau_{n}(M(J)) \leqslant 3$ by Corollary 4.9, and the conclusion follows from Definition 4.3.

Interchanging the roles of $M$ and $M^{\prime}$, we see that the second inequality in (4.4) amounts to showing that $\tau_{n}\left(M^{\prime}(J)\right) \leqslant 2$ for each non-empty set $J \in[\mathbb{N}]^{<\infty}$ with $M(J) \in \mathcal{S}_{n}$. We shall establish this estimate by induction on $n \in \mathbb{N}_{0}$.

Note that we start the induction at $n=0$ for convenience. Indeed, the estimate is clear in this case because the non-empty $\mathcal{S}_{0}$-sets are precisely the singletons, so in fact $M(J) \in \mathcal{S}_{0}$ implies that $M^{\prime}(J) \in \mathcal{S}_{0}$.

Now assume inductively that we have established the estimate for some $n \in \mathbb{N}_{0}$, and let $J \in[\mathbb{N}]^{<\infty}$ be a non-empty set with $M(J) \in \mathcal{S}_{n+1}$. Set $j=\min J$. Then, by the definition of $\mathcal{S}_{n+1}$, we can find $h \in \mathbb{N}$ and $J_{1}<J_{2}<\cdots<J_{h}$ such that $h \leqslant m_{j}, J=\bigcup_{i=1}^{h} J_{i}$ and $M\left(J_{i}\right) \in \mathcal{S}_{n}$ for each $i \in\{1, \ldots, h\}$.

Take $k \in \mathbb{N}$ and $L_{1}<L_{2}<\cdots<L_{k}$ such that $J=\bigcup_{i=1}^{k} L_{i}, M^{\prime}\left(L_{i}\right)$ is a maximal $\mathcal{S}_{n}$-set for each $i \in\{1, \ldots, k-1\}$ and $M^{\prime}\left(L_{k}\right) \in \mathcal{S}_{n}$. The induction hypothesis implies that $k \leqslant 2 h$ because, by Remark 4.2(ii), each of the sets $J_{i}$ can be split into at most two successive pieces $J_{i}^{\prime}<J_{i}^{\prime \prime}$ with $M^{\prime}\left(J_{i}^{\prime}\right), M^{\prime}\left(J_{i}^{\prime \prime}\right) \in \mathcal{S}_{n}$. If $k \leqslant m_{j}^{\prime}=\min M^{\prime}(J)$, then $M^{\prime}(J) \in \mathcal{S}_{n+1}$, and the conclusion follows, so we may suppose that $k>m_{j}^{\prime}$. We observe that $m_{j}^{\prime} \geqslant j$ because $m_{j}^{\prime}$ is the $j^{\text {th }}$ element of a strictly increasing sequence of natural numbers, and hence $E:=\bigcup_{i=1}^{j} M^{\prime}\left(L_{i}\right) \in \mathcal{S}_{n+1}$. Since the sets $L_{1}, \ldots, L_{j+1}$ are successive and non-empty, we see that $\min L_{j+1} \geqslant \min L_{1}+j=2 j$, so that $\min M^{\prime}\left(L_{j+1}\right) \geqslant m_{2 j}^{\prime}=2 m_{j} \geqslant 2 h \geqslant k$, which implies that $F:=\bigcup_{i=j+1}^{k} M^{\prime}\left(L_{i}\right) \in \mathcal{S}_{n+1}$. This shows that $\tau_{n+1}\left(M^{\prime}(J)\right) \leqslant 2$ because $M^{\prime}(J)=E \cup F$, and hence the induction continues.

The final clause is immediate from Corollary 4.5.

Proof of Proposition 4.6. Let $M \in[\mathbb{N}]$, and set $M^{\prime}=(2 M-1) \cup(2 M) \in[\mathbb{N}]$. Then $X_{M} \cong X_{M^{\prime}}$ by Lemma 4.11, and $X_{M^{\prime}} \cong X_{2 M-1} \oplus X_{2 M}$ because $M^{\prime}$ is the disjoint union of the sets $2 M-1$ and $2 M$. Hence the result will follow provided that we can show that $X_{2 M-1}$ and $X_{2 M}$ are both isomorphic to $X_{M}$.

The map $\sigma: j \mapsto 2 j, \mathbb{N} \rightarrow \mathbb{N}$, is strictly increasing, and the corresponding left shift $L_{\sigma}$ is a left inverse of the right shift $R$, using the notation of Lemma 4.7 and Corollary 4.9. Hence the restriction of $R$ to $X_{M}$ is an isomorphism onto its image, which is $X_{2 M}$, with the inverse being the appropriate restriction of $L_{\sigma}$.

A similar argument using the strictly increasing map $\sigma: j \mapsto 2 j-1, \mathbb{N} \rightarrow \mathbb{N}$, and the right shift given by $\sum_{j} \alpha_{j} e_{j} \mapsto \sum_{j} \alpha_{j} e_{2 j-1}$ (which is bounded because it equals the composition $L_{\rho} \circ R$, where $\rho: j \mapsto j+1, \mathbb{N} \rightarrow \mathbb{N}$, and $R$ is given by (4.3) as above) shows that $X_{M} \cong X_{2 M-1}$. 
Using these results, we obtain the following characterization of when two spatial ideals of $\mathscr{B}\left(X\left[\mathcal{S}_{n}\right]\right)$ are equal. It is the counterpart of Corollary 3.2 and will play a similar role in our proof of Lemma 2.8 for the Schreier spaces of finite order.

Proposition 4.12. Let $X=X\left[\mathcal{S}_{n}\right]$ for some $n \in \mathbb{N}$, and suppose that $M, N \in[\mathbb{N}]$ satisfy $P_{M} \in \overline{\left\langle P_{N}\right\rangle}$. Then the following conditions are equivalent:

(a) $P_{N} \in \overline{\left\langle P_{M}\right\rangle}$;

(b) $\left\langle P_{M}\right\rangle=\left\langle P_{N}\right\rangle$;

(c) $X_{M}$ is isomorphic to $X_{N}$;

(d) $X_{N}$ is isomorphic to a subspace of $X_{M}$;

(e) the $n^{\text {th }}$ Gasparis-Leung index $d_{n}(N, M)$ is finite;

(f) there is a constant $k \in \mathbb{N}$ such that $\tau_{n}(N(J)) \leqslant k$ for each set $J \in[\mathbb{N} \cap(k, \infty)]^{<\infty}$ with $M(J) \in \mathcal{S}_{n}$.

Proof. Conditions (a) and (b) are equivalent by Lemma 2.1, while Proposition 4.6 implies that Corollary 2.5 applies, and therefore conditions (b) and (c) are also equivalent.

Condition (c) trivially implies (d), which in turn implies (e) by Theorem 4.4. Combining the assumption that $P_{M} \in \overline{\left\langle P_{N}\right\rangle}$ with Lemma 2.3 and Proposition 4.6, we deduce that $X_{N}$ contains a complemented subspace which is isomorphic to $X_{M}$, and therefore $d_{n}(M, N)$ is finite by Theorem 4.4. Thus the implication $(\mathrm{e}) \Rightarrow(\mathrm{c})$ follows from Corollary 4.5.

Definition 4.3 shows that (e) implies (f). Conversely, suppose that $k \in \mathbb{N}$ is a constant such that (f) is satisfied. Then, for each set $J \in[\mathbb{N}]^{<\infty}$ with $M(J) \in \mathcal{S}_{n}$, the subadditivity of $\tau_{n}$ stated in (4.1) implies that

$$
\tau_{n}(N(J)) \leqslant \tau_{n}(N(J \cap[1, k]))+\tau_{n}(N(J \cap(k, \infty))) \leqslant \tau_{n}(N(\{1, \ldots, k\}))+k,
$$

and therefore $d_{n}(N, M) \leqslant \tau_{n}(N(\{1, \ldots, k\}))+k<\infty$.

Lemma 4.13. Let $M, N \in[\mathbb{N}]$, and suppose that $J \in[\mathbb{N}]<\infty$ is a non-empty set such that $N \cap[1, \max M(J)) \subseteq M$. Then $N(J)$ is a spread of $M(J)$.

Proof. Write $M=\left\{m_{1}<m_{2}<\cdots\right\}$ and $N=\left\{n_{1}<n_{2}<\cdots\right\}$, and let $j \in J$. We must show that $m_{j} \leqslant n_{j}$. This is clear if $n_{j} \geqslant \max M(J)$ because $m_{j} \in M(J)$. Otherwise $n_{j} \in N \cap[1, \max M(J))$, which is contained in $M$ by the assumption, so that $n_{j}=m_{k}$ for some $k \in \mathbb{N}$. We have $k \geqslant j$ because $n_{1}, \ldots, n_{j-1} \in\left\{m_{1}, \ldots, m_{k-1}\right\}$, and therefore $m_{j} \leqslant m_{k}=n_{j}$, as required.

Proof of Lemma 2.8 for $X=X\left[\mathcal{S}_{n}\right], n \in \mathbb{N}$. (i). Let $N \in[\mathbb{N}]$, and let $\left(F_{j}^{n}(N)\right)_{j \in \mathbb{N}}$ be the unique sequence of successive, maximal $\mathcal{S}_{n}$-sets partitioning $N$ described on page 13 . The fact that the sets $F_{1}^{n}(N), F_{2}^{n}(N), \ldots$ are successive and partition $N$ means that we can partition $\mathbb{N}$ into successive intervals $J_{1}<J_{2}<\cdots$ such that $F_{j}^{n}(N)=N\left(J_{j}\right)$ for each $j \in \mathbb{N}$, where $N\left(J_{j}\right)$ (unlike $F_{j}^{n}(N)$ ) is defined using the notation (4.2). Set $k_{1}=1$ and recursively define $k_{j+1}=k_{j}+j$ for $j \in \mathbb{N}$. (In other words, $k_{j+1}=j(j+1) / 2+1$, but this formula is not helpful for our purposes). Then, setting $K_{j}=\bigcup_{i=k_{j}}^{k_{j+1}-1} J_{i} \in[\mathbb{N}]^{<\infty}$ for each 
$j \in \mathbb{N}$, we obtain a partition of $\mathbb{N}$ into successive intervals such that

$$
N\left(K_{j}\right)=\bigcup_{i=k_{j}}^{k_{j+1}-1} F_{i}^{n}(N) .
$$

Thus $N\left(K_{j}\right)$ is the union of $k_{j+1}-k_{j}=j$ successive, maximal $\mathcal{S}_{n}$-sets, so $\tau_{n}\left(N\left(K_{j}\right)\right)=j$.

Since $n \geqslant 1, N$ contains arbitrarily long $\mathcal{S}_{n}$-sets. This fact enables us to recursively choose successive intervals $L_{1}<L_{2}<\cdots$ in $\mathbb{N}$ such that $\left|L_{j}\right|=\left|K_{j}\right|$ and $N\left(L_{j}\right) \in \mathcal{S}_{n}$ for each $j \in \mathbb{N}$. Indeed, once the intervals $L_{1}<\cdots<L_{j}$ have been chosen for some $j \in \mathbb{N}$, we can take $\ell>\max L_{j}$ so large that the interval $L_{j+1}=\left[\ell, \ell+\left|K_{j}\right|\right) \cap \mathbb{N}$ satisfies $N\left(L_{j+1}\right) \in \mathcal{S}_{n}$, and hence the recursion continues.

Set $L=\bigcup_{j \in \mathbb{N}} L_{j} \in[\mathbb{N}]$, and observe that $L\left(K_{j}\right)=L_{j}$ for each $j \in \mathbb{N}$ because $\left(K_{j}\right)$ is a partition of $\mathbb{N}$ into successive intervals with $\left|K_{j}\right|=\left|L_{j}\right|$. Consequently $M:=N(L) \in[N]$ satisfies

$$
M\left(K_{j}\right)=(N(L))\left(K_{j}\right)=N\left(L\left(K_{j}\right)\right)=N\left(L_{j}\right) \in \mathcal{S}_{n},
$$

so $d_{n}(N, M) \geqslant \tau_{n}\left(N\left(K_{j}\right)\right)=j$. Since this is true for every $j \in \mathbb{N}$, Proposition 4.12 implies that $P_{N} \notin\left\langle P_{M}\right\rangle$.

(ii). Let $M \subseteq N$ be infinite subsets of $\mathbb{N}$ with $P_{N} \notin\left\langle P_{M}\right\rangle$. By Corollary 2.4, we may suppose that $\min M=\min N$ by adding the element $\min N$ to the set $M$ if necessary. In order to define a map $\varphi: \mathscr{P}(\mathbb{N}) \rightarrow[N]$ which satisfies conditions (III.i)-(III.iii) in Lemma 2.7, we shall construct a sequence $\left(J_{i}\right)_{i \in \mathbb{N}}$ of finite, successive intervals of $\mathbb{N}$ such that:

(i) $\bigcup_{i \in \mathbb{N}} J_{i}=\mathbb{N}$;

(ii) for each $j \in \mathbb{N}, J_{j}$ contains a subset $K_{j}$ such that $\tau_{n}\left(N\left(K_{j}\right)\right) \geqslant j$ and the set

$$
L_{j}=M \cup \bigcup_{i<j} N\left(J_{i}\right) \in[N]
$$

satisfies $L_{j}\left(K_{j}\right) \in \mathcal{S}_{n}$ and $L_{j}\left(K_{j}\right) \subseteq N\left(J_{j}\right)$.

The construction is by recursion, where condition (i) is replaced with the appropriate finite analogue, that is,

(i') $\bigcup_{i=1}^{j} J_{i}=\mathbb{N} \cap\left[1, \max J_{j}\right]$ for each $j \in \mathbb{N}$.

To begin the recursion, we define $J_{1}=K_{1}=\{1\}$. Then (i') is obvious, and (ii) follows almost as easily because $L_{1}=M$ by definition and $M\left(K_{1}\right)=\{\min M\}=N\left(J_{1}\right)$; being a singleton, this set belongs to $\mathcal{S}_{n}$.

Now assume recursively that, for some $j \in \mathbb{N}$, we have chosen finite, successive intervals $J_{1}<\cdots<J_{j}$ such that conditions (i') and (ii) are satisfied. Following (4.5), we define $L_{j+1}=M \cup \bigcup_{i \leqslant j} N\left(J_{i}\right)$. Then $L_{j+1} \backslash M$ is finite, so Corollary 2.4 implies that

$$
\left\langle P_{L_{j+1}}\right\rangle=\left\langle P_{M}\right\rangle \not P_{N} .
$$

Hence, by Proposition 4.12 , we can find a set $K_{j+1} \in\left[\mathbb{N} \cap\left(\max J_{j}, \infty\right)\right]^{<\infty}$ such that $L_{j+1}\left(K_{j+1}\right) \in \mathcal{S}_{n}$ and $\tau_{n}\left(N\left(K_{j+1}\right)\right)>\max J_{j}$. We see that $\max J_{j} \geqslant j$ because the sets $J_{1}, \ldots, J_{j}$ are non-empty and successive, and consequently $\tau_{n}\left(N\left(K_{j+1}\right)\right) \geqslant j+1$. Since 
$L_{j+1} \subseteq N$, we can choose $k \in \mathbb{N}$ such that $\max L_{j+1}\left(K_{j+1}\right)$ is the $k^{\text {th }}$ element of $N$. Note that $k \geqslant \max K_{j+1}$, so that $J_{j+1}=\mathbb{N} \cap\left(\max J_{j}, k\right]$ is a finite successor interval of $J_{j}$ containing $K_{j+1}$ and such that $\left(\mathrm{i}^{\prime}\right)$ is satisfied for $j+1$. Moreover, $\max L_{j+1}\left(K_{j+1}\right) \in N\left(J_{j+1}\right)$ by the choice of $k$, while

$$
L_{j+1}\left(K_{j+1}\right)>L_{j+1}\left(\bigcup_{i \leqslant j} J_{i}\right)=N\left(\bigcup_{i \leqslant j} J_{i}\right),
$$

so $L_{j+1}\left(K_{j+1}\right) \subseteq N\left(J_{j+1}\right)$ because $N\left(J_{j+1}\right)$ is the immediate successor interval of $N\left(\bigcup_{i \leqslant j} J_{i}\right)$ in $N$. This shows that (ii) is also satisfied for $j+1$, and hence the recursion continues.

For $D \subseteq \mathbb{N}$, set $D^{c}=\mathbb{N} \backslash D$, and define $\varphi: \mathscr{P}(\mathbb{N}) \rightarrow[N]$ by

$$
\varphi(D)=M \cup \bigcup_{i \in D^{c}} N\left(J_{i}\right)
$$

This map clearly satisfies conditions (III.i)-(III.ii) in Lemma 2.7. To help us establish condition (III.iii), we shall show that

$$
\varphi(D) \cap\left[1, \max L_{j}\left(K_{j}\right)\right) \subseteq L_{j} \quad(D \in \mathscr{P}(\mathbb{N}), j \in D),
$$

where $L_{j}$ is given by (4.5). Indeed, suppose that $k \in \varphi(D) \cap\left[1, \max L_{j}\left(K_{j}\right)\right)$ for some $j \in D \subseteq \mathbb{N}$. Since $M \subseteq L_{j}$, it suffices to consider the case where $k \in N\left(J_{i}\right)$ for some $i \in D^{c}$. We must have $i \leqslant j$ because

$$
k<\max L_{j}\left(K_{j}\right) \leqslant \max N\left(J_{j}\right) .
$$

Moreover, $i \neq j$ because $i \in D^{c}$ and $j \in D$. Hence $i<j$, so $L_{j} \supseteq N\left(J_{i}\right) \ni k$, as desired.

Now let $D \subseteq \mathbb{N}$. Suppose first that $P_{N} \in\left\langle P_{\varphi(D)}\right\rangle$, and let $j \in D$. Combining Lemma 4.13 with (4.7), we see that $(\varphi(D))\left(K_{j}\right)$ is a spread of $L_{j}\left(K_{j}\right)$, so that $(\varphi(D))\left(K_{j}\right) \in \mathcal{S}_{n}$, and therefore $d_{n}(N, \varphi(D)) \geqslant \tau_{n}\left(N\left(K_{j}\right)\right) \geqslant j$. Thus the set $D$ is bounded above by $d_{n}(N, \varphi(D))$, which is finite by Proposition 4.12. Hence $D$ is finite. Conversely, suppose that $D$ is finite. Then $N \backslash \varphi(D) \subseteq \bigcup_{i \in D} N\left(J_{i}\right)$ is also finite, and therefore $P_{N} \in\left\langle P_{\varphi(D)}\right\rangle$ by Corollary 2.4. This completes the proof of (III.iii) and hence of the first bullet point in Lemma 2.8(ii).

To establish the second, let $\Gamma=\left\{\overline{\left\langle P_{L_{j}}\right\rangle}: j \in \mathbb{N}\right\}$ be a set-induced chain in $\Omega_{M, N}$, where $M \subseteq L_{j} \subseteq L_{j+1} \subseteq N$ and $P_{N} \notin \overline{\left\langle P_{L_{j}}\right\rangle}$ for each $j \in \mathbb{N}$. We shall recursively choose non-empty, finite subsets $J_{1}, J_{2}, \ldots$ of $\mathbb{N}$ such that

$$
\tau_{n}\left(N\left(J_{j}\right)\right) \geqslant j, \quad L_{j}\left(J_{j}\right) \in \mathcal{S}_{n} \quad \text { and } \quad L_{j+1}\left(J_{j+1}\right)>L_{j}\left(J_{j}\right) \quad(j \in \mathbb{N}) .
$$

We begin this recursion by taking $J_{1}=\{1\}$. Now assume that non-empty, finite subsets $J_{1}, \ldots, J_{j}$ of $\mathbb{N}$ have been chosen for some $j \in \mathbb{N}$, and take $k \in \mathbb{N}$ such that $\max L_{j}\left(J_{j}\right)$ is the $k^{\text {th }}$ element of $L_{j+1}$. Since $P_{N} \notin \overline{\left\langle P_{L_{j+1}}\right\rangle}$, Proposition 4.12 enables us to choose a set $J_{j+1} \in[\mathbb{N} \cap(k, \infty)]^{<\infty}$ such that $\tau_{n}\left(N\left(J_{j+1}\right)\right)>j$ and $L_{j+1}\left(J_{j+1}\right) \in \mathcal{S}_{n}$. Then the first two statements in (4.8) are satisfied for $j+1$, while the last part follows from the fact that

$$
L_{j}\left(J_{j}\right) \subseteq L_{j+1}(\mathbb{N} \cap[1, k])<L_{j+1}\left(J_{j+1}\right) .
$$

Hence the recursion continues. 
Set $\ell_{0}=0$ and $\ell_{j}=\max L_{j}\left(J_{j}\right)$ for $j \in \mathbb{N}$, and define $L=\bigcup_{j \in \mathbb{N}} L_{j} \cap\left(\ell_{j-1}, \ell_{j}\right]$. We check that $\overline{\left\langle P_{L}\right\rangle} \in \Omega_{M, N}$ :

- each $m \in M$ belongs to $\left(\ell_{j-1}, \ell_{j}\right]$ for some $j \in \mathbb{N}$, and also $m \in L_{j}$ because $M \subseteq L_{j}$, so $m \in L$;

- $L \subseteq N$ because $L_{j} \subseteq N$ for each $j \in \mathbb{N}$;

- for each $j \in \mathbb{N}$, we have

$$
L \cap\left[1, \ell_{j}\right]=\bigcup_{i=1}^{j} L_{i} \cap\left(\ell_{i-1}, \ell_{i}\right] \subseteq L_{j},
$$

so Lemma 4.13 implies that $L\left(J_{j}\right)$ is a spread of $L_{j}\left(J_{j}\right)$, and therefore $L\left(J_{j}\right) \in \mathcal{S}_{n}$; hence $d_{n}(N, L) \geqslant \tau_{n}\left(N\left(J_{j}\right)\right) \geqslant j$, so as $j \in \mathbb{N}$ was arbitrary, we conclude that $P_{N} \notin \overline{\left\langle P_{L}\right\rangle}$ by Proposition 4.12 .

We observe that $L_{j} \cap\left(\ell_{j-1}, \infty\right) \subseteq L$ for each $j \in \mathbb{N}$ because the sequence $\left(L_{j}\right)$ is increasing. This implies that $P_{L_{j}} \in\left\langle P_{L}\right\rangle$ by Corollary 2.4 , and thus $\overline{\left\langle P_{L}\right\rangle}$ is an upper bound for $\Gamma$, as desired.

Lemma 4.14. For each $n \in \mathbb{N}$, the formal identity operator from $X\left[\mathcal{S}_{n}\right]$ to $c_{0}$ is a strictly singular, non-compact operator of norm one.

Proof. Set $X=X\left[\mathcal{S}_{n}\right]$, and let $S: \operatorname{span}\left\{e_{j}: j \in \mathbb{N}\right\} \rightarrow c_{0}$ be the formal identity operator, that is, the linear map determined by $S e_{j}=d_{j}$ for each $j \in \mathbb{N}$, where $\left(d_{j}\right)$ denotes the unit vector basis for $c_{0}$. Writing $\left(e_{j}^{*}\right)$ for the coordinate functionals in $X^{*}$ corresponding to the basis $\left(e_{j}\right)$ for $X$, we observe that

$$
\|S x\|_{\infty}=\sup \left\{\left|\left\langle x, e_{j}^{*}\right\rangle\right|: j \in \mathbb{N}\right\}
$$

for each $x \in \operatorname{span}\left\{e_{j}: j \in \mathbb{N}\right\}$, and therefore $S$ is bounded with norm 1, so it extends uniquely to an operator defined on all of $X$, also denoted by $S$ and still of norm 1 ; for later reference, we note that (4.9) remains valid for each $x \in X$. This operator $S$ cannot be compact because $\left(e_{j}\right)$ is a bounded sequence in $X$ such that no subsequence of $\left(S e_{j}\right)$ converges in $c_{0}$.

Assume towards a contradiction that $S$ is not strictly singular. Then $X$ contains a closed, infinite-dimensional subspace $W$ such that there exists an $\varepsilon>0$ for which $\|S w\|_{\infty} \geqslant \varepsilon\|w\|_{X}$ for each $w \in W$. Choose $m \in \mathbb{N} \cap\left[2(1+\varepsilon) / \varepsilon^{2}, \infty\right)$, and set $k_{1}=m$. We can then recursively choose numbers $k_{2}, \ldots, k_{m+1}$ with $k_{j+1}>k_{j}$ and unit vectors $w_{j} \in W \cap \overline{\operatorname{span}}\left\{e_{i}: i \geqslant k_{j}\right\}$ such that $\left|\left\langle w_{j}, e_{i}^{*}\right\rangle\right| \leqslant \varepsilon / m$ for each $i \geqslant k_{j+1}$ and $j \in\{1, \ldots, m\}$.

Set $w=\sum_{j=1}^{m} w_{j} \in W$. We claim that $\left|\left\langle w, e_{i}^{*}\right\rangle\right| \leqslant 1+\varepsilon$ for each $i \in \mathbb{N}$. There are three cases to examine:

- The estimate is obvious for $i<k_{1}$ because $\left\langle w, e_{i}^{*}\right\rangle=0$ for such $i$.

- Suppose that $i \in\left[k_{j}, k_{j+1}\right)$ for some $j \in\{1, \ldots, m\}$. Then $\left\langle w_{h}, e_{i}^{*}\right\rangle=0$ for $h>j$, so

$$
\left|\left\langle w, e_{i}^{*}\right\rangle\right| \leqslant \sum_{h=1}^{j-1}\left|\left\langle w_{h}, e_{i}^{*}\right\rangle\right|+\left|\left\langle w_{j}, e_{i}^{*}\right\rangle\right| \leqslant \frac{(j-1) \varepsilon}{m}+\left\|w_{j}\right\|_{X} \leqslant \varepsilon+1 .
$$


- Finally, for $i \geqslant k_{m+1},\left|\left\langle w_{j}, e_{i}^{*}\right\rangle\right| \leqslant \varepsilon / m$ for each $j \in\{1, \ldots, m\}$, so $\left|\left\langle w, e_{i}^{*}\right\rangle\right| \leqslant \varepsilon$. This establishes the claim, and consequently $\|S w\|_{\infty} \leqslant 1+\varepsilon$ by (4.9).

For each $j \in\{1, \ldots, m\}$, we have $\left\|S w_{j}\right\|_{\infty} \geqslant \varepsilon$, so another application of (4.9) enables us to choose $h_{j} \in \mathbb{N}$ such that $\left|\left\langle w_{j}, e_{h_{j}}^{*}\right\rangle\right| \geqslant \varepsilon$. We note that necessarily $h_{j} \in\left[k_{j}, k_{j+1}\right)$, and therefore the set $\left\{h_{1}, h_{2}, \ldots, h_{m}\right\}$ belongs to $\mathcal{S}_{1}$ and thus to $\mathcal{S}_{n}$. This implies that

$$
\begin{aligned}
\|w\|_{X} & \geqslant \sum_{i=1}^{m}\left|\left\langle w, e_{h_{i}}^{*}\right\rangle\right|=\sum_{i=1}^{m}\left|\left\langle\sum_{j=1}^{i} w_{j}, e_{h_{i}}^{*}\right\rangle\right| \\
& \geqslant \sum_{i=1}^{m}\left(\left|\left\langle w_{i}, e_{h_{i}}^{*}\right\rangle\right|-\sum_{j=1}^{i-1}\left|\left\langle w_{j}, e_{h_{i}}^{*}\right\rangle\right|\right) \geqslant \sum_{i=1}^{m}\left(\varepsilon-\frac{(i-1) \varepsilon}{m}\right)=\frac{(m+1) \varepsilon}{2} .
\end{aligned}
$$

Combining the above estimates, we conclude that

$$
1+\varepsilon \geqslant\|S w\|_{\infty} \geqslant \varepsilon\|w\|_{X} \geqslant \frac{(m+1) \varepsilon^{2}}{2},
$$

which contradicts that we chose $m \geqslant 2(1+\varepsilon) / \varepsilon^{2}$.

Proof of Theorem 1.2(ii). As Odell [31, p. 694] observed, the space $X=X\left[\mathcal{S}_{n}\right]$ is $c_{0}$-saturated (in the sense that each of its closed, infinite-dimensional subspaces contains an isomorphic copy of $c_{0}$ ) because $X$ embeds into $C\left[0, \omega^{\omega^{n}}\right]$, which is $c_{0}$-saturated. Sobczyk's Theorem implies that every copy of $c_{0}$ in $X$ is automatically complemented, so that $X$ is subprojective, and therefore $\mathscr{S}(X)=\mathscr{E}(X)$ by Pfaffenberger's result [33].

Let $S: X \rightarrow c_{0}$ be the formal identity operator, as in the proof of Lemma 4.14 above. Since $X$ contains a complemented copy of $c_{0}$, we can choose operators $U: c_{0} \rightarrow X$ and $V: X \rightarrow c_{0}$ such that $I_{c_{0}}=V U$. Then $U S \in \mathscr{S}(X) \backslash \mathscr{K}(X)$ because $S \in \mathscr{S}\left(X, c_{0}\right)$ and $V(U S)=S \notin \mathscr{K}\left(X, c_{0}\right)$ by Lemma 4.14, and consequently $\mathscr{S}(X) \neq \mathscr{K}(X)$.

Finally, let $Q \in \mathscr{B}(X)$ be a projection whose image is isomorphic to $c_{0}$. Then $Q \notin \mathscr{S}(X)$. However, for each non-trivial spatial ideal $\mathscr{I}$, say $\mathscr{I}=\overline{\left\langle P_{N}\right\rangle}$, where $N \in[\mathbb{N}]$, we can factor $Q$ through $X_{N}$ because $X$ is $c_{0}$-saturated, and therefore $Q \in \mathscr{I}$. This shows that $Q$ belongs to the intersection on the left-hand side of (1.1), and the conclusion follows.

\section{Some open questions}

Theorem 1.2(ii) and its proof raise some natural questions. To state them concisely, let $X=X\left[\mathcal{S}_{n}\right]$ for some $n \in \mathbb{N}$, and denote the closure of the ideal of operators on $X$ which factor through $c_{0}$ by $\overline{\mathscr{G}}_{c_{0}}(X)$; in the notation of the proof of Theorem 1.2(ii), $\overline{\mathscr{G}}_{c_{0}}(X)=\overline{\langle Q\rangle}$, and the argument given in its last paragraph shows that

$$
\overline{\mathscr{G}}_{c_{0}}(X) \subseteq \bigcap\{\mathscr{I}: \mathscr{I} \text { is a non-trivial spatial ideal of } \mathscr{B}(X)\} .
$$

However, we do not know whether this inclusion is proper. We also do not know whether $\mathscr{S}(X) \subseteq \overline{\mathscr{G}}_{c_{0}}(X)$.

Another, somewhat less precise, question is as follows. It applies to both $X=T$ and $X=X\left[\mathcal{S}_{n}\right]$ for $n \in \mathbb{N}$. Theorem 1.1 (iii) states that $\mathscr{B}(X)$ contains at least continuum 
many maximal ideals, but we do not have an explicit description of a single such ideal. We know that they cannot be spatial, but is it possible to describe at least some of these maximal ideals explicitly?

Acknowledgements. The research presented in this paper was initiated when the thirdand second-named authors visited Washington \& Lee University, VA, in October 2015 and 2016, respectively, supported by Washington \& Lee Summer Lenfest grants. It was continued when the first-named author visited the UK in February/March 2017, supported by a Scheme 2 grant from the London Mathematical Society. Kania's work has also received funding from GAČR project 19-07129Y; RVO 67985840 (Czech Republic). We gratefully acknowledge this support. Finally, we would like to thank the referee for their careful reading of our paper, especially for suggesting what is now stated as the first part of Lemma 2.7 as a way of simplifying the proof of the second part of that lemma.

\section{REFERENCES}

[1] D. E. Alspach and S. Argyros, Complexity of weakly null sequences, Diss. Math. (Rozprawy Mat.) 321 (1992).

[2] S. A. Argyros, K. Beanland and P. Motakis, Strictly singular operators in Tsirelson like spaces, Illinois J. Math. 57 (2013), 1173-1217.

[3] S. A. Argyros and R. G. Haydon, A hereditarily indecomposable $\mathscr{L}_{\infty}$-space that solves the scalarplus-compact problem, Acta Math. 206 (2011), 1-54.

[4] E. Berkson and H. Porta, Representations of $\mathfrak{B}(X)$, J. Funct. Anal. 3 (1969), 1-34.

[5] J. W. Calkin, Two-sided ideals and congruences in the ring of bounded operators in Hilbert space, Ann. of Math. 42 (1941), 839-873.

[6] P. G. Casazza, W. B. Johnson and L. Tzafriri, On Tsirelson's space, Israel J. Math. 47 (1984), 81-98.

[7] P. G. Casazza and T. J. Shura, Tsirelson's space. Lecture Notes in Mathematics 1363. Springer-Verlag, Berlin, 1989.

[8] H. G. Dales, R. J. Loy and G. A. Willis, Homomorphisms and derivations from $\mathscr{B}(E)$, J. Funct. Anal. 120 (1994), 201-219.

[9] M. Daws, Closed ideals in the Banach algebra of operators on classical non-separable spaces, Math. Proc. Cambridge Philos. Soc. 140 (2006), 317-332.

[10] T. Figiel, An example of infinite dimensional reflexive Banach space non-isomorphic to its Cartesian square, Studia Math. 42 (1972), 295-306.

[11] T. Figiel and W. B. Johnson, A uniformly convex Banach space which contains no $\ell_{p}$, Compos. Math. 29 (1974), 179-190.

[12] D. Freeman, Th. Schlumprecht and A. Zsák, Closed ideals of operators between the classical sequence spaces, Bull. Lond. Math. Soc. 49 (2017), 859-876.

[13] I. Gasparis, A dichotomy theorem for subsets of the power set of the natural numbers, Proc. Amer. Math. Soc. 129 (2001), 759-764.

[14] I. Gasparis and D. H. Leung, On the complemented subspaces of the Schreier spaces, Studia Math. 141 (2000), 273-300.

[15] I. C. Gohberg, A. S. Markus and I. A. Feldman, Normally solvable operators and ideals associated with them, Amer. Math. Soc. Translat. 61 (1967), 63-84, Russian original in Bul. Akad. Śtiince RSS Moldoven 10 (76) (1960), 51-70.

[16] M. González, A. Martínez-Abejón and M. Salas-Brown, Perturbation classes for semi-Fredholm operators on subprojective and superprojective spaces, Ann. Acad. Sci. Fenn. Math. 36 (2011) 481-491. 
[17] B. Gramsch, Eine Idealstruktur Banachscher Operatoralgebren, J. Reine Angew. Math. 225 (1967), 97-115.

[18] W. B. Johnson, G. Pisier and G. Schechtman, Ideals in L(L $)$, Math. Ann. 376 (2020), 693-705.

[19] W. B. Johnson and G. Schechtman, The number of closed ideals in $L\left(L_{p}\right)$, preprint available at arXiv:2003.11414.

[20] T. Kania and T. Kochanek, The ideal of weakly compactly generated operators acting on a Banach space, J. Operator Th. 71 (2014), 455-477.

[21] T. Kania and N. J. Laustsen, Ideal structure of the algebra of bounded operators acting on a Banach space, Indiana Univ. Math. J. 66 (2017), 1019-1043.

[22] D. Kleinecke, Almost-finite, compact, and inessential operators, Proc. Amer. Math. Soc. 14 (1963), 863-868.

[23] P. Koszmider, On decompositions of Banach spaces of continuous functions on Mrówka's spaces, Proc. Amer. Math. Soc. 133 (2005), 2137-2146.

[24] P. Koszmider and N. J. Laustsen, A Banach space induced by an almost disjoint family, admitting only few operators and decompositions, preprint available at arXiv:2003.03832.

[25] N. J. Laustsen and R. J. Loy, Closed ideals in the Banach algebra of operators on a Banach space, in Proceedings of the Conference on Topological Algebras, their Applications, and Related Topics (ed. K. Jarosz and A. Sołtysiak), Banach Center Publications 67 (2005), 245-264.

[26] N. J. Laustsen, R. J. Loy and C. J. Read, The lattice of closed ideals in the Banach algebra of operators on certain Banach spaces, J. Funct. Anal. 214, (2004), 106-131.

[27] N. J. Laustsen, Th. Schlumprecht and A. Zsák, The lattice of closed ideals in the Banach algebra of operators on a certain dual Banach space, J. Operator Th. 56 (2006), 391-402.

[28] E. Luft, The two-sided closed ideals of the algebra of bounded linear operators of a Hilbert space, Czechoslovak Math. J. 18 (1968), 595-605.

[29] P. Mankiewicz, A superreflexive Banach space $X$ with $L(X)$ admitting a homomorphism onto the Banach algebra $C(\beta \mathbf{N})$, Israel J. Math. 65 (1989), 1-16.

[30] P. Motakis, D. Puglisi and D. Zisimopoulou, A hierarchy of separable commutative Calkin algebras, Indiana Univ. Math. J. 65 (2016), 39-67.

[31] E. Odell, On quotients of Banach spaces having shrinking unconditional bases, Illinois J. Math. 36 (1992), 681-695.

[32] A. Pełczyński, On the isomorphism of the spaces $m$ and M, Bull. Acad. Pol. Sci. 6 (1958), 695-696.

[33] W. Pfaffenberger, On the ideals of strictly singular and inessential operators, Proc. Amer. Math. Soc. 25 (1970), 603-607.

[34] A. Pietsch, Operator ideals, North Holland, 1980.

[35] H. Porta, Two sided ideals of operators, Bull. Amer. Math. Soc. 75 (1969), 599-602.

[36] H. Porta, Factorable and strictly singular operators. I, Studia Math. 37 (1970/71), 237-243.

[37] Th. Schlumprecht and A. Zsák, The algebra of bounded linear operators on $\ell_{p} \oplus \ell_{q}$ has infinitely many closed ideals, J. Reine Angew. Math. 735 (2018), 225-247.

[38] G. Sirotkin and B. Wallis, Sequence-singular operators, J. Math. Anal. Appl. 443 (2016), 1208-1219.

[39] M. Tarbard, Hereditarily indecomposable, separable $\mathscr{L}_{\infty}$ Banach spaces with $\ell_{1}$ dual having few but not very few operators, J. London Math. Soc. 85 (2012), 737-764.

[40] B. S. Tsirelson, It is impossible to imbed $\ell_{p}$ or $c_{0}$ into an arbitrary Banach space (in Russian), Funktsional. Anal. i Prilozhen 8 (1974), 57-60.

[41] P. Volkmann, Operatoralgebren mit einer endlichen Anzahl von maximalen Idealen, Studia Math. 55 (1976), 151-156.

[42] B. Wallis, Closed ideals in $\mathscr{L}(X)$ and $\mathscr{L}\left(X^{*}\right)$ when $X$ contains certain copies of $\ell_{p}$ and $c_{0}$, Oper. Matrices 10 (2016), 285-318. 
(K. Beanland) Department of Mathematics, Washington and Lee University, Lexington, VA 24450, USA

Email address: beanlandk@wlu.edu

(T. Kania) Mathematical Institute, Czech Academy of Sciences, Žitná 25, 11567 Praha 1, Czech Republic; and Institute of Mathematics and Computer Science, Jagiellonian UniVERSity, ŁoJASIEWICZA 6, 30-348 Kraków, POland

Email address: kania@math.cas.cz, tomasz.marcin.kania@gmail.com

(N. J. Laustsen) Department of Mathematics and Statistics, Fylde College, Lancaster University, LANCASTER, LA1 4YF, United Kingdom

Email address: n.laustsen@lancaster.ac.uk 